\title{
Generalized wave-front reconstruction algorithm applied in a Shack-Hartmann test
}

\author{
Weiyao Zou and Zhenchao Zhang
}

\begin{abstract}
A generalized numerical wave-front reconstruction method is proposed that is suitable for diversified irregular pupil shapes of optical systems to be measured. That is, to make a generalized and regular normal equation set, the test domain is extended to a regular square shape. The compatibility of this method is discussed in detail, and efficient algorithms (such as the Cholesky method) for solving this normal equation set are given. In addition, the authors give strict analyses of not only the error propagation in the wave-front estimate but also of the discretization errors of this domain extension algorithm. Finally, some application examples are given to demonstrate this algorithm. (C) 2000 Optical Society of America
\end{abstract}

OCIS codes: $\quad 010.7350,100.5070,120.6650,220.4840$.

\section{Introduction}

Recently, the Shack-Hartmann (S-H) wave-front test, a technique developed for analyzing aberrations of optical systems, has become widely used in optical shop testing and telescopes, especially in active optics and adaptive optics. The measurement data of a $\mathrm{S}-\mathrm{H}$ test are the coordinate differences of the $\mathrm{S}-\mathrm{H}$ grid that contain wave-front slope information. Therefore a numerical reconstruction procedure is necessary for integrating these slope data to obtain the desired wave-front map. Generally, this wavefront estimate procedure is laborious and time consuming, especially for a large $\mathrm{S}-\mathrm{H}$ sampling point array with nonstandard pupil outlines. Each estimation approach may be categorized as being either zonal or modal, depending on whether the estimate is a phase value in a local zone or a coefficient of an aperture function. ${ }^{1}$ The modal estimate method usually uses Zernike or Legendre polynomials or

\footnotetext{
When this research was performed, the authors were with the Nanjing Astronomical Instruments Research Center, Chinese Academy of Sciences and Astronomical Optics Laboratory, National Astronomical Observatories, Chinese Academy of Sciences, Nanjing 210042, China. W. Zou (zou@gps.caltech.edu) is now a visitor with the division of Geological and Planetary Sciences, MS 150-21, California Institute of Technology, Pasadena, California 91125. Z. Zhang's e-mail address is zczhang@nairc.ac.cn.

Received 3 March 1999; revised manuscript received 14 July 1999.

0003-6935/00/020250-19\$15.00/0

(C) 2000 Optical Society of America
}

complex exponentials as expansion functions. ${ }^{2}$ Therefore, restricted by the orthogonality requirements of polynomials employed, it is difficult to adopt the modal estimation to generally irregular arrays of sampling points. In contrast, the zonal estimate method does not have the orthogonality restrictions, and it is applicable to the irregular as well as the regular sampling arrays. However, because of the irregularity of the integration boundaries, we will encounter different equation sets in zonal estimation with different aperture shapes. For the smaller S-H sampling arrays it is a simpler task, but it is a much more difficult, laborious, and time-consuming task for a large array of sampling points, such as the case of a large-aperture astronomical telescope. Therefore, to make optical testing (especially in an optical shop) more efficient, a generalized wave-front reconstruction algorithm that is accommodated to arbitrary aperture shapes is necessary and deserves to be studied in greater depth.

Many wave-front reconstruction algorithms have been reported in previous studies. ${ }^{1-14}$ These algorithms are of either zonal estimation or modal estimation or a combination of both in a least-squares sense, and are thus applicable to slope-sensitive tests, such as a shearing interferometer and a S-H test. In this paper the authors introduce a zonal least-squares-fit algorithm of general purpose, which is different from previous ones but also applicable to $\mathrm{S}-\mathrm{H}$ testing as well as to shearing interferometry. 


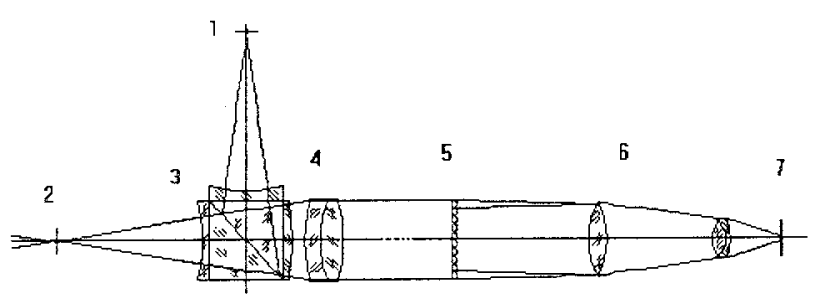

Fig. 1. Schematic configuration of the optical system of the $\mathrm{S}-\mathrm{H}$ test. 1, Pinhole reference source; 2 , focus of the optical system to be tested; 3 , beam-splitter cube; 4 , collimator; 5 , lenslet array; 6 , reducing system; 7 , CCD target.

\section{Numerical Integral Method in Wave-Front Reconstruction}

The principle of the $\mathrm{S}-\mathrm{H}$ wave-front test is shown schematically in Fig. $1 .^{15}$ In Fig. 1, point 1 is the reference-point light source and point 2 is the focus of the optical system to be tested. A beam of light from point 1 passes through the beam-splitter cube (3), the collimator (4), and the lenslet array (5) and then forms an array of the $\mathrm{S}-\mathrm{H}$ grid, which is imaged onto the CCD focal plane by the reducing system (6). This array on the CCD is used as the $\mathrm{S}-\mathrm{H}$ standard grid array. Alternately, another beam of light from the optical system to be measured passes through the same beam-splitter cube, the collimator, the lenslet array, and the reducing system; then it is imaged onto the CCD focal plane as the $\mathrm{S}-\mathrm{H}$ measurement grid array. The coordinate differences between the two grid arrays are written as $\left(l_{y i}, l_{z i}\right)$; the relationship between wave-front aberration and ray aberration is shown in Fig. 2. The exit pupil shown in Fig. 2 is at the position of the lenslet array, and $f_{0}$ is the focal length of the lenslet array. $\left[y_{i}^{(0)}, z_{i}^{(0)}\right](i=1$, $2, \ldots, N$, where $i$ denotes the serial number of subapertures of the lenslet array) denote the coordinates of the S-H standard grid points, and $\left[y_{i}^{(1)}, z_{i}^{(1)}\right](i=1$, $2, \ldots, N)$ denote the coordinates of the $\mathrm{S}-\mathrm{H}$ measurement grid points; so we have

$$
\begin{aligned}
& l_{y i}=y_{i}^{(1)}-y_{i}^{(0)}, \\
& l_{z i}=z_{i}^{(1)}-z_{i}^{(0)} .
\end{aligned}
$$

There is a sufficiently accurate approximate formula for most practical purposes, ${ }^{10}$

$$
\begin{aligned}
& \left.\frac{\partial \mathbf{W}}{\partial y}\right|_{i}=\frac{l_{y i}}{f_{0}}, \\
& \left.\frac{\partial \mathbf{W}}{\partial z}\right|_{i}=\frac{l_{z i}}{f_{0}}, i=1,2, \ldots, N,
\end{aligned}
$$

where $\mathbf{W}$ is the wave-front aberration to be measured. Therefore the gradient of the wave front is

$$
\operatorname{grad} \mathbf{W}=\frac{\partial \mathbf{W}}{\partial y} i+\frac{\partial \mathbf{W}}{\partial z} j=\mathbf{f}_{1}(y, z) i+\mathbf{f}_{2}(y, z) j .
$$

Let us postulate that the values of $\partial \mathbf{W} / \partial y$ and $\partial \mathbf{W} / \partial z$ at each set of measurement grid points are given and that the gradients (slopes) between two adjacent



Fig. 2. Schematics of the relationship between wave-front aberration and ray aberration in the $\mathrm{S}-\mathrm{H}$ test.

measurement grid points can be linearly interpolated.14 Considering that the lenslet array is equidistant, in the $y$ direction (as shown in Fig. 3), we have

$$
\frac{\partial \mathbf{W}}{\partial y}=\left.\frac{\partial \mathbf{W}}{\partial y}\right|_{i}\left(1-\frac{y}{a}\right)+\left.\frac{\partial \mathbf{W}}{\partial y}\right|_{i+1} \frac{y}{a},
$$

where $a$ is the distance between two adjacent grid points. Integrating this partial differential equation, we obtain

$$
\mathbf{W}_{i+1}-\mathbf{W}_{i}=\frac{1}{2}\left(\left.\frac{\partial \mathbf{W}}{\partial y}\right|_{i}+\left.\frac{\partial \mathbf{W}}{\partial y}\right|_{i+1}\right) a .
$$

The S-H grid points can be divided into two groups: interior grid points and boundary grid points. The interior grid points have four adjacent points, and the boundary grid points have fewer than four adjacent points. For an interior point, we get

$$
\begin{aligned}
& w_{i}-w_{i-1}=\frac{1}{2}\left(\left.\frac{\partial \mathbf{W}}{\partial y}\right|_{i}+\left.\frac{\partial \mathbf{W}}{\partial y}\right|_{i-1}\right) a, \\
& w_{i+1}-w_{i}=\frac{1}{2}\left(\left.\frac{\partial \mathbf{W}}{\partial y}\right|_{i+1}+\left.\frac{\partial \mathbf{W}}{\partial y}\right|_{i}\right) a, \\
& w_{i-t}-w_{i}=\frac{1}{2}\left(\left.\frac{\partial \mathbf{W}}{\partial z}\right|_{i-t}+\left.\frac{\partial \mathbf{W}}{\partial z}\right|_{i}\right) a, \\
& w_{i}-w_{i+t}=\frac{1}{2}\left(\left.\frac{\partial \mathbf{W}}{\partial z}\right|_{i}+\left.\frac{\partial \mathbf{W}}{\partial z}\right|_{i+t}\right) a .
\end{aligned}
$$

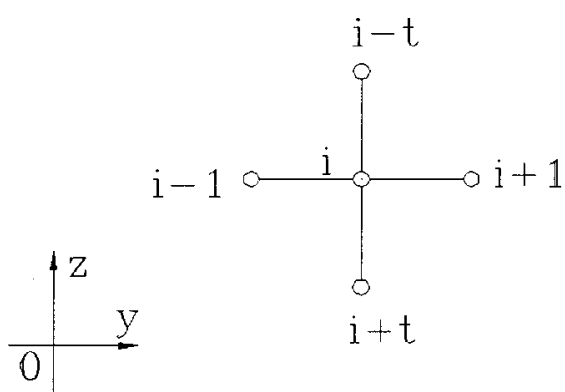

Fig. 3. $\mathrm{S}-\mathrm{H}$ grid at point $i$, where point $i$ is an interior grid point. It is similar to the cross difference scheme of partial differential equations. 
For each boundary point we can obtain two or three equations that are similar to Eqs. (2.6). In 1986, Su et al. proposed a zonal integral method for wave-front reconstruction (published in 1992; see Ref. 14) that is based on the assumption that slopes are linear functions of coordinates between each set of sampling points. The assumption found in Ref. 14 is equivalent to the assumption used by Ghozeil. ${ }^{11}$ The difference between the studies of Su et al. and of Ghozeil is that Ghozeil employed a numerical integration method to get the discrete values of wave-front aberrations, whereas Su et al. translated the wavefront reconstruction integration into solution of a linear equation set. Su et al. pointed out that the slope changes between adjacent points could be assumed to be nonlinear functions as well as linear functions, depending on specified situations we encountered. For simplicity, Su et al. took the linear assumption. Just before the revision of the present paper, Zou found that Southwell had obtained a similar formulation of Eqs. (2.6). ${ }^{1}$ Nevertheless, $\mathrm{Su}$ et al. proposed this assumption and obtained these equations independently. The current paper is based on the studies of Su et al. which are described in detail below.

According to Eqs. (2.6), the problem of solving elliptic partial differential equations is transformed into the problem of the least-squares solution of a linear equation set. This least-squares solution is unique to within an additive constant. For astronomical applications this equation set is usually large and sparse. For example, the $\mathrm{S}-\mathrm{H}$ system in the New Technology Telescope developed at the European Southern Observatory has 700 grid points, and the $\mathrm{S}-\mathrm{H}$ system in the first experimental system of thin-mirror active optics developed at the Nanjing Astronomical Instruments Research Center (NAIRC) has 161 grid points. The iterative method can be used to solve the corresponding normal equations, which avoids the computermemory problem that the direct solution method may encounter.

\section{Establishment of Generalized Wave-Front Reconstruction Equations}

Generally, astronomical telescopes have central obstructions of the secondary and the supporting spiders, and the optical components to be tested in an optical shop are of various kinds of contour shape. This brings complexities to wave-front reconstruction in practice: much repetitive research must be done, because we have to study the corresponding solution methods according to different pupil types. So developing a generalized and efficient wave-front reconstruction algorithm is necessary.

It is known that the coefficient matrix of an equation set for wave-front reconstruction is closely related to the shape of the sampling array and its serial numbering. To make the coefficient matrix regular

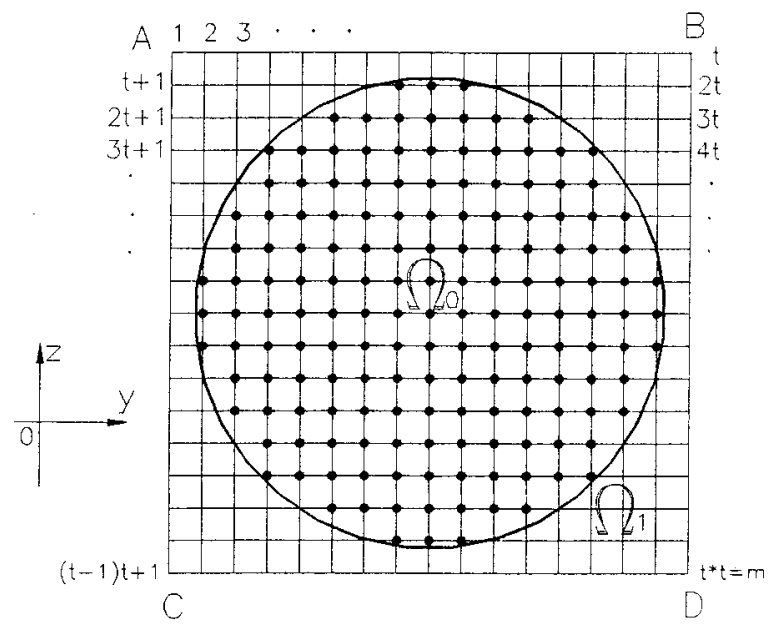

Fig. 4. Extended S-H grid array. Circular domain $\boldsymbol{\Omega}_{0}$ is the Ø500-mm thin mirror (with 6-mm thickness) of the active optics experiment system in the NAIRC. There are $161 \mathrm{~S}-\mathrm{H}$ grid points in $\boldsymbol{\Omega}_{0}$. After domain extension, $\boldsymbol{\Omega}_{0}$ becomes a $15 \times 15$ (or $17 \times 17$ ) square grid mesh $\boldsymbol{\Omega}_{1}$, which has 225 (or 289) grip points.

and simple, the following important improvements are adopted (as shown in Fig. 4):

1. Fill the four outer corners of the testing domain (exit pupil) $\boldsymbol{\Omega}_{0}$ and extend it to a regular square net $\boldsymbol{\Omega}_{1}$, which covers the whole exit pupil $\boldsymbol{\Omega}_{0}$.

2. Index the grid points in $\boldsymbol{\Omega}_{1}$ serially from 1 to $m$ row by row (or column by column, alternatively), and all the integrations are made along the positive directions of the $y$ and the $z$ axes.

3. Let the slopes be zero at the grid points in the additive extended regions $\boldsymbol{\Omega}_{1} \backslash \boldsymbol{\Omega}_{0}$.

To preserve its universality, we assume that this regular square net has $t \times t=m$ grid points. Then from Eqs. (2.6) we have

in the $y$ direction,

$$
w_{i+1}-w_{i}=g_{i, i+1}, \quad i \neq k t, \quad k=1,2, \ldots, t
$$

in the $z$ direction,

$$
w_{i}-w_{i+t}=f_{i, i+t}, \quad i=1,2, \ldots, m-t,
$$

where

$$
\begin{aligned}
g_{i, i+1} & =\frac{1}{2}\left(\left.\frac{\partial \mathbf{W}}{\partial y}\right|_{i+1}+\left.\frac{\partial \mathbf{W}}{\partial y}\right|_{i}\right) a, \\
f_{i, i+t} & =\frac{1}{2}\left(\left.\frac{\partial \mathbf{W}}{\partial z}\right|_{i}+\left.\frac{\partial \mathbf{W}}{\partial z}\right|_{i+t}\right) a .
\end{aligned}
$$

Thus 


$$
\begin{array}{rlrl}
w_{2}-w_{1} & =g_{2,1}, & w_{1}-w_{1+t}=f_{1,1+t}, \\
w_{3}-w_{2}=g_{3,2}, & w_{2}-w_{2+t}=f_{2,2+t}, \\
\cdots & \cdots & \\
w_{t}-w_{t-1}=g_{t, t-1}, & w_{t-1}-w_{2 t-1}=f_{t-1,2 t-1}, \\
w_{t}-w_{2 t}=f_{t, 2 t}, \\
w_{t+2}-w_{t+1}=g_{+2 t, t+1}, & w_{t+1}-w_{2 t+1}=f_{t+1,2 t+1}, \\
\cdots & \\
w_{m-t}-w_{m-t-1} & =g_{m-t, m-t-1}, & \\
w_{m-t+2}-w_{m-t+1} & =g_{m-t+2, m-t+1}, & \\
\cdots &
\end{array}
$$

A total of $2(t-1)=2 m-2 t$ equations can be obtained. When we write them in matrix form, we get

$$
\mathbf{A W}=\mathbf{F}
$$

or

$$
\begin{aligned}
& {\left[\begin{array}{ccccc}
-1 & 1 & & & \\
& -1 & 1 & & \\
& & \ddots & \ddots & \\
& & & -1 & 1
\end{array}\right.} \\
& -1 \quad 1 \\
& \begin{array}{lll}
-1 & 1 & \\
& & \ddots
\end{array} \\
& -11 \\
& \begin{array}{llllll}
1 & 0 & 0 & \cdots & 0 & -1
\end{array} \\
& \begin{array}{ccccccc}
1 & 0 & 0 & \cdots & 0 & -1 & \\
& \ddots & \ddots & \ddots & & \ddots & \ddots
\end{array}
\end{aligned}
$$



Then the normal equation set of these overdetermined linear equations can be written as

$$
\mathbf{A}^{T} \mathbf{A W}=\mathbf{A}^{T} \mathbf{F},
$$

where $\mathbf{A} \in \mathfrak{R}^{2 m(m-t) \times m}, \mathbf{A}=[\mathbf{B} / \mathbf{C}], \mathbf{W} \in \mathfrak{R}^{m \times 1}$, and $\mathbf{F}=[\mathbf{g} / \mathbf{f}] \in \mathfrak{R}^{2(m-t) \times 1}$, where $\mathbf{B}=\operatorname{diag}\left[\mathbf{D}_{1}, \mathbf{D}_{2}, \ldots\right.$, $\left.\mathbf{D}_{t}\right] \in \mathfrak{R}^{(m-t) \times m}$ and $\mathbf{C}=\mathfrak{R}^{(m-t) \times m}$ is a sparse band matrix, where

$$
\mathbf{D}_{i}=\left[\begin{array}{ccccc}
-1 & 1 & & & \\
& -1 & 1 & & \\
& & \ddots & \ddots & \\
& & & -1 & 1
\end{array}\right]_{(t-1) \times t}, \quad i=1,2, \ldots, t .
$$

where

$$
\mathbf{A}^{T} \mathbf{A}=\mathbf{B}^{T} \mathbf{B}+\mathbf{C}^{T} \mathbf{C}
$$

where

$$
\mathbf{B}^{T} \mathbf{B}=\operatorname{diag}\left[\mathbf{D}_{1}{ }^{T} \mathbf{D}_{1}, \mathbf{D}_{2}{ }^{T} \mathbf{D}_{2}, \ldots, \mathbf{D}_{t}^{T} \mathbf{D}_{t}\right]_{m \times m},
$$


where

$$
\mathbf{D}_{i}^{T} \mathbf{D}_{i}=\left[\begin{array}{cccccc}
1 & -1 & & & & \\
-1 & 2 & -1 & & & \\
& \ddots & \ddots & \ddots & & \\
& & & -1 & 2 & -1 \\
& & & & -1 & 1
\end{array}\right]_{t \times t}
$$

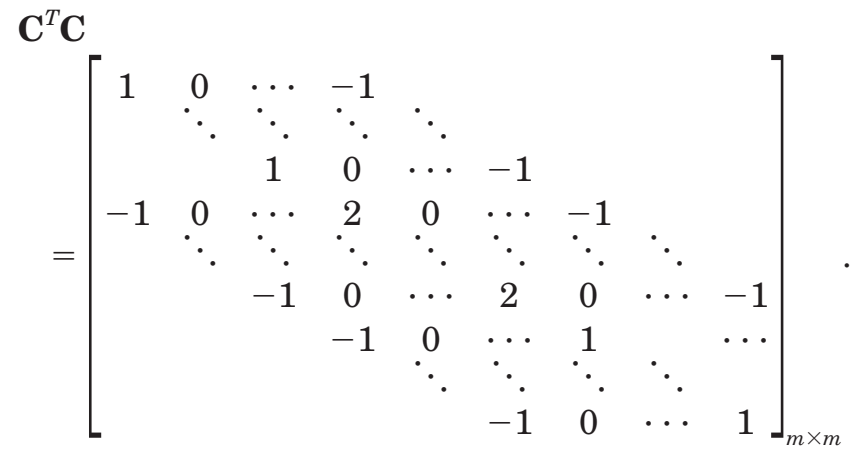

Hence

$$
\mathbf{A}^{T} \mathbf{A}=\left[\begin{array}{ccccc}
\mathbf{E}_{1} & -\mathbf{I} & & & \\
-\mathbf{I} & \mathbf{E}_{2} & -\mathbf{I} & & \\
& \ddots & \ddots & \ddots & \\
& & -\mathbf{I} & \mathbf{E}_{2} & -\mathbf{I} \\
& & & -\mathbf{I} & \mathbf{E}_{1}
\end{array}\right]_{m \times m}
$$

where

$$
\begin{aligned}
\mathbf{E}_{1}= & {\left[\begin{array}{ccccc}
2 & -1 & & & \\
-1 & 3 & -1 & & \\
& \ddots & \ddots & \ddots & \\
& & -1 & 3 & -1 \\
& & & -1 & 2
\end{array}\right]_{t \times t}, } \\
\mathbf{E}_{2}= & {\left[\begin{array}{ccccc}
3 & -1 & & & \\
-1 & 4 & -1 & & \\
& \ddots & \ddots & \ddots & \\
& & -1 & 4 & -1 \\
& & & -1 & 3
\end{array}\right]_{t \times t}, } \\
& -\mathbf{I}=\left[\begin{array}{llll}
-1 & \ddots & \\
& & -1
\end{array}\right]_{t \times t} .
\end{aligned}
$$

In Appendix A it is demonstrated that

$$
\operatorname{rank}\left(\mathbf{A}^{T} \mathbf{A}\right)=\operatorname{rank}(\mathbf{A})=m-1
$$

Moreover,

$$
\mathbf{A}^{T} \mathbf{F}=\mathbf{B}^{T} g+\mathbf{C}^{T} f=\left[\begin{array}{l}
f_{1, t+1}-g_{2,1} \\
f_{2, t+2}+g_{2,1}-g_{3,2} \\
\vdots \\
f_{t-1,2 t-1}+g_{t-1, t-2}-g_{t, t-1} \\
f_{t, 2 t}+g_{t, t-1} \\
f_{t+1,2 t+1}-f_{1, t+1}-g_{t+2, t+1} \\
\vdots \\
f_{i, i+1}-f_{i-t, i}+g_{i, i-1}-g_{i+1, i} \\
\vdots \\
f_{m, m-t}-f_{m-2 t, m-t}+g_{m-t, m-t-1} \\
\vdots \\
-f_{m-t, m}+g_{m, m-1}
\end{array}\right] .
$$

According to these normal equations, for each interior grid point $w_{i}$, we get

$$
\begin{array}{r}
-w_{i-t}-w_{i-1}+4 w_{i}-w_{i+1}-w_{i-t} \\
=f_{i, i+t}-f_{i-1, i}+g_{i, i-1}-g_{i+1, i},
\end{array}
$$

or

$$
\begin{aligned}
w_{i}= & \frac{1}{4}\left(w_{i-t}+w_{i-1}+w_{i+1}+w_{i+t}\right) \\
& +\frac{a}{8}\left(\left.\frac{\partial \mathbf{W}}{\partial y}\right|_{i-1}+\left.\frac{\partial \mathbf{W}}{\partial z}\right|_{i+t}-\left.\frac{\partial \mathbf{W}}{\partial z}\right|_{i-t}-\left.\frac{\partial \mathbf{W}}{\partial y}\right|_{i+1}\right) .
\end{aligned}
$$

Likewise, we can write out the corresponding equation of each boundary grid, such as $i=1, t, m-t+$ 1. For instance,

$$
\begin{aligned}
w_{1}= & \frac{1}{2}\left(w_{2}+w_{t+1}\right)+\frac{a}{4}\left(\left.\frac{\partial \mathbf{W}}{\partial z}\right|_{1}+\left.\frac{\partial \mathbf{W}}{\partial z}\right|_{t+1}\right. \\
& \left.-\left.\frac{\partial \mathbf{W}}{\partial y}\right|_{2}-\left.\frac{\partial \mathbf{W}}{\partial y}\right|_{1}\right), \\
w_{t}= & \frac{1}{2}\left(w_{t-1}+w_{2 t}\right)+\frac{a}{4}\left(\left.\frac{\partial \mathbf{W}}{\partial z}\right|_{t}+\left.\frac{\partial \mathbf{W}}{\partial z}\right|_{2 t}\right. \\
& \left.+\left.\frac{\partial \mathbf{W}}{\partial y}\right|_{t}+\left.\frac{\partial \mathbf{W}}{\partial y}\right|_{t-1}\right) .
\end{aligned}
$$

From Eqs. (3.18)-(3.20) we find that the relationship between the actual phases of the wave fronts and their partial derivatives is quite lucid in the leastsquares sense. We can obtain these equations simply by performing four operations with Eqs. (2.6). The wave-front aberration at each grid point (interior or boundary) is only directly related to the wave-front aberrations and partial derivatives of its adjacent grid points. This also indicates that the equations for boundary points have nothing to do with the contour shape and the size of their integral domains but do have some differences between the line-boundary points and corner-boundary points.

After this paper was submitted for publication, Zou later noticed that Hunt had obtained similar matrix 
equations. ${ }^{7}$ However, Hunt's formulations are restricted to a square phase array, but the formulations developed in this paper are generalized to fit moregeneral situations.

\section{Compatibility of Domain Extension}

Substantially, the problem of determining the phase of a wave front from its gradients (partial derivatives) is a Neumann problem of Poission's equation. Assume that the gradient function of a measured wave front is

$$
\mathbf{f}_{0}(y, z)=\mathbf{f}_{1}(y, z) i+\mathbf{f}_{2}(y, z) j .
$$

It includes the real wave-front gradient function $\nabla \mathbf{W}$ and the measurement noise function $\mathbf{n}(y, z)$; so

$$
\mathbf{f}_{0}(y, z)=\nabla \mathbf{W}+\mathbf{n}(y, z) .
$$

In a least-squares sense, minimizing the functional

$$
\iint\left[\nabla \mathbf{W}-\mathbf{f}_{0}(y, z)\right]^{2} \mathrm{~d} y \mathrm{~d} z
$$

is the extreme-value problem of a functional.

Let

$$
\begin{aligned}
\mathbf{F}\left(y, z, \mathbf{W}_{y}, \mathbf{W}_{z}\right) & =\left[\nabla \mathbf{W}-\mathbf{f}_{0}(y, z)\right]^{2} \\
& =\left[\frac{\partial \mathbf{W}}{\partial y} i+\frac{\partial \mathbf{W}}{\partial z} j-\mathbf{f}_{0}(y, z)\right]^{2} .
\end{aligned}
$$

We get a functional

$$
\mathbf{J}[\mathbf{W}(y, z)]=\iint_{\Omega} \mathbf{F}\left(y, z, \mathbf{W}_{y}, \mathbf{W}_{z}\right) \mathrm{d} y \mathrm{~d} z .
$$

Therefore its permissible function set is

$$
\mathbf{S}=\left\{\mathbf{W}\left|\mathbf{W} \in \mathbf{C}^{1}(\overline{\mathbf{\Omega}}), \frac{\partial \mathbf{W}}{\partial \mathbf{n}}\right|_{\partial \Omega}=\mathbf{g}(y, z)\right\} .
$$

We can derive its Euler's equation, which is

$$
\frac{\partial}{\partial y}\left(\frac{\partial \mathbf{F}}{\partial \mathbf{W}_{y}}\right)+\frac{\partial}{\partial z}\left(\frac{\partial \mathbf{F}}{\partial \mathbf{W}_{z}}\right)=0 .
$$

Hence

$$
\frac{\partial^{2} \mathbf{W}}{\partial y^{2}}+\frac{\partial^{2} \mathbf{W}}{\partial z^{2}}=i \frac{\partial \mathbf{f}_{0}}{\partial y}+j \frac{\partial \mathbf{f}_{0}}{\partial z} .
$$

Then we rewrite it as

$$
\nabla^{2} \mathbf{W}=\nabla \mathbf{f}_{0}=\mathbf{f}(y, z),
$$

where $\mathbf{W}$ is an estimation of the real wave front in least-squares sense. Equation (4.9) is a Poission equation. We therefore propose the following proposition about the Neumann boundary-value problem of Eq. (4.9) in the extended domain.

In $\mathfrak{R}^{2}$, assume that $\boldsymbol{\Omega}_{0}$ is a compositum whose boundary is a piecewise smooth curve $\partial \boldsymbol{\Omega}_{0}$, and $\mathbf{C}_{0}$ is the outer boundary of $\boldsymbol{\Omega}_{0}$; therefore $\partial \boldsymbol{\Omega}_{0}=\mathbf{C}_{0}+$ $\cup_{i=1}^{n} \mathbf{C}_{i}$, where $\mathbf{C}_{i}$ is the boundary of $\mathbf{D}_{i}, \mathbf{D}_{i} \not \subset \mathbf{\Omega}_{0}$, the

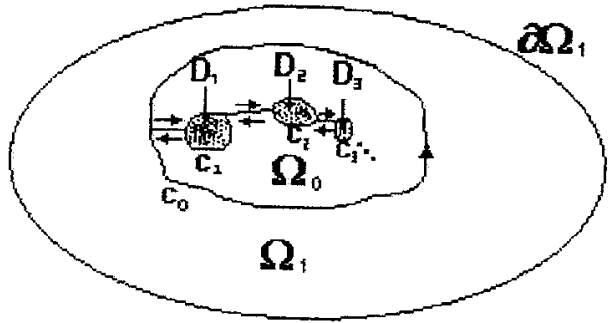

Fig. 5. Compositum $\boldsymbol{\Omega}_{0}$ and the extended $\boldsymbol{\Omega}_{0}, \boldsymbol{\Omega}_{1}, \mathbf{D}_{1}, \mathbf{D}_{2}, \mathbf{D}_{3}$, etc., are the blind areas with boundaries $\mathbf{C}_{1}, \mathbf{C}_{2}, \mathbf{C}_{3}$, etc., in $\boldsymbol{\Omega}_{0} . \mathbf{C}_{0}$ is the outer boundary of $\boldsymbol{\Omega}_{0}$, and $\partial \boldsymbol{\Omega}_{0}$ is the outer boundary of $\boldsymbol{\Omega}_{0}$.

function $\mathbf{W}$ together with its first and second derivatives are continuous on the closed region $\overline{\boldsymbol{\Omega}}_{0}$, and

$$
\left\{\begin{array}{l}
\nabla^{2} \mathbf{W}=\mathbf{f}(y, z), \quad(y, z) \in \mathbf{\Omega}_{0} \\
\left.\frac{\partial \mathbf{W}}{\partial \mathbf{n}}\right|_{\partial \Omega_{0}}=\mathbf{g}(y, z)
\end{array} ;\right.
$$

then its solution exists, and it is unique except for an additive constant. ${ }^{16}$ Clearly, the method of Su et al . and other previous methods are the numerical solutions to this problem.

Now we discuss the compatibility of the domain extension we have made. Consider the Neumann boundary problem of the Poission equation, assuming that an arbitrary $\boldsymbol{\Omega}_{1}$ (shown in Fig. 5) is given, $\boldsymbol{\Omega}_{0} \subseteq$ $\boldsymbol{\Omega}_{1}$, and let all the partial derivatives be zero in the additive extended regions:

$$
\left.\left.\frac{\partial \mathbf{W}}{\partial y}\right|_{\Omega_{1} \Omega_{0}} \equiv \frac{\partial \mathbf{W}}{\partial z}\right|_{\Omega_{1} \Omega_{0}} \equiv 0 .
$$

Then clearly the extended $\mathbf{W}$ cannot satisfy the derivative continuity condition on $\partial \boldsymbol{\Omega}_{0}$; so the domain extension is not strictly compatible in theory. However, this compatibility can be approximately satisfied, and the domain extension is feasible in practice. Actually, we know the wave-front gradients only in domain $\boldsymbol{\Omega}_{0}$, as shown in Fig. 6. For grid points inside $\boldsymbol{\Omega}_{0}, w_{i}$ can be expressed as in formula (3.18). For grid points on the boundary of $\boldsymbol{\Omega}_{0}$, take point 40

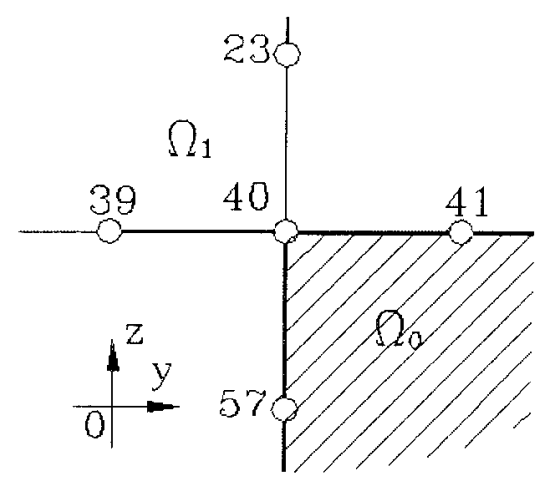

Fig. 6. Point 40 is a corner-boundary grid point in $\boldsymbol{\Omega}_{0}$. Points 40 , 41 , and 57 are $\mathrm{S}-\mathrm{H}$ grid points in $\boldsymbol{\Omega}_{0}$; points 23 and point 39 are the invented grid points in $\boldsymbol{\Omega}_{1} \backslash \boldsymbol{\Omega}_{0}$. 
as an example (shown in Fig. 6); according to the normal equation (3.6), we obtain

$$
\begin{aligned}
w_{40}= & \frac{1}{4}\left(w_{23}+w_{57}+w_{41}+w_{39}\right)+\frac{a}{8}\left(\left.\frac{\partial \mathbf{W}}{\partial y}\right|_{39}+\left.\frac{\partial \mathbf{W}}{\partial z}\right|_{57}\right. \\
& \left.-\left.\frac{\partial \mathbf{W}}{\partial z}\right|_{23}-\left.\frac{\partial \mathbf{W}}{\partial y}\right|_{41}\right) .
\end{aligned}
$$

Consider

$$
\left.\frac{\partial \mathbf{W}}{\partial y}\right|_{39}=0,\left.\quad \frac{\partial \mathbf{W}}{\partial z}\right|_{23}=0
$$

therefore

$$
w_{23}-w_{40}=\frac{1}{2}\left(\left.\frac{\partial \mathbf{W}}{\partial z}\right|_{40}+\left.\frac{\partial \mathbf{W}}{\partial z}\right|_{23}\right) a=\frac{1}{2}\left(\left.\frac{\partial \mathbf{W}}{\partial z}\right|_{40}\right) a .
$$

We have

$$
w_{23}=w_{40}+\frac{1}{2}\left(\left.\frac{\partial \mathbf{W}}{\partial z}\right|_{40}\right) a \text {. }
$$

Since

$$
w_{40}-w_{39}=\frac{1}{2}\left(\left.\frac{\partial \mathbf{W}}{\partial y}\right|_{40}+\left.\frac{\partial \mathbf{W}}{\partial y}\right|_{39}\right) a=\frac{1}{2}\left(\left.\frac{\partial \mathbf{W}}{\partial y}\right|_{40}\right) a,
$$

we get

$$
w_{39}=w_{40}-\frac{1}{2}\left(\left.\frac{\partial \mathbf{W}}{\partial y}\right|_{40}\right) a .
$$

Applying Eqs. (4.13) and (4.14) into Eq. (4.12), we obtain

$$
\begin{aligned}
w_{40}= & \frac{1}{2}\left(w_{57}+w_{41}\right)+\frac{a}{4}\left(\left.\frac{\partial \mathbf{W}}{\partial z}\right|_{40}+\left.\frac{\partial \mathbf{W}}{\partial z}\right|_{57}\right. \\
& \left.-\left.\frac{\partial \mathbf{W}}{\partial y}\right|_{40}-\left.\frac{\partial \mathbf{W}}{\partial y}\right|_{41}\right) .
\end{aligned}
$$

Comparing Eq. (4.15) with Eq. (3.19), we find that, if points 23 and 39 do not exist, then point 40 is a corner point of $\boldsymbol{\Omega}_{0}$. The situation of point 40 is similar to that of point 1 , which is a corner point of $\boldsymbol{\Omega}_{1}$. The wave aberration expressions at the two points are identical. However, $w_{23}$ and $w_{39}$, which satisfy Eq. (3.7), do not strictly satisfy Eq. (4.13) and Eq. (4.14); so Eq. (4.15) is only an approximate equation. The above analyses are also applicable to other boundary points of $\boldsymbol{\Omega}_{0}$. Customarily, precision requirements of the optical surface in fringe parts are slightly lower than those of the interior parts. For convenience of discussion we let $\mathbf{W}_{0}$ denote the reconstructed wave front in $\boldsymbol{\Omega}_{0}$ before domain extension, and $\mathbf{W}_{1}$ denotes the reconstructed wave front in $\boldsymbol{\Omega}_{1}$ (the extended $\boldsymbol{\Omega}_{0}$ ) after domain extension. Therefore, since the partial derivatives of $\mathbf{W}_{1}$ on $\partial \boldsymbol{\Omega}_{0}$ are not continuous, the smoothing effect of the numerical solution usually makes $\mathbf{W}_{1}$ in $\boldsymbol{\Omega}_{0}$ not identical to $\mathbf{W}_{0}$. The differences are detectable mainly in the boundary parts of $\mathbf{W}_{0}$. However, it can be demonstrated in examples (see

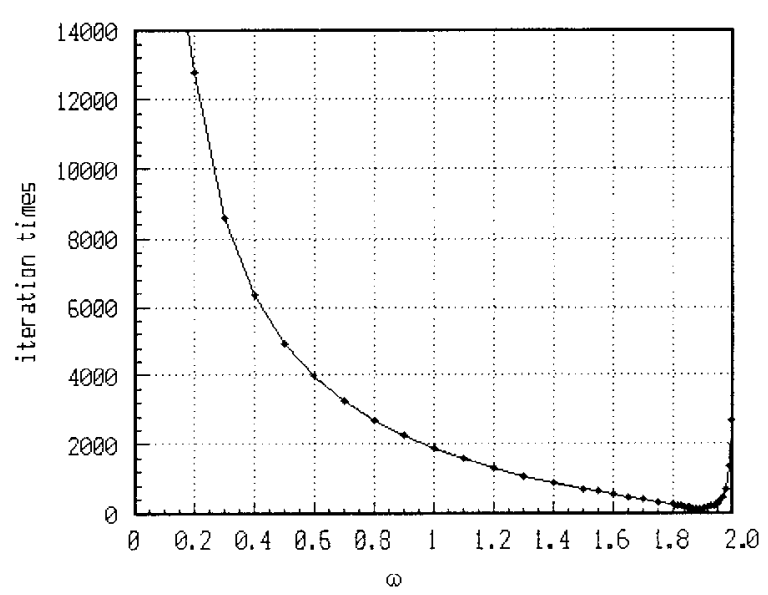

Fig. 7. Relationship between the relaxation factor $\omega$ and the corresponding iteration times for convergence. It indicates that the optimal relaxation factor is 1.881 and the corresponding iteration time for convergence is 111 .

Figs. 11-14 below) that these discrepancies are so negligible that they can be ignored, and the extension method is virtually feasible. Summarily, by extending the real pupil boundary to a regular outline, we can simplify the wave-front reconstruction algorithm and transform the problem of irregular boundary into a problem of regular boundary.

After this paper was submitted for publication, Zou later noted that Roddier and Roddier ${ }^{9}$ and Freischlad ${ }^{13}$ once separately introduced similar extension algorithms that are suited for arbitrary pupil shapes. But the methods in Refs. 9 and 13 are based on the Fourier transform; thus they are actually different from the extension algorithm described in this paper.

\section{Solutions of the Normal Equations}

\section{A. Iterative Method}

The rank of the normal equation set is $m-1$; so we should add a constraint to the normal equation set to make it of full rank, that is, to determine the zero point of the reconstructed wave front. Actually, we need not add an additional row to $\mathbf{A}^{T} \mathbf{A}$ as Southwell did. ${ }^{1}$ This task can be achieved by addition of a large constant $\left(\sim 10^{10}\right)$ to the diagonal element at whose point the phase value of the wave front will be zero, ${ }^{19}$ and we still keep the form of coefficient matrix $\mathbf{A}^{T} \mathbf{A}$ unchanged.

For this kind of large sparse matrix equation, iterative methods, such as the Jacobi method, the Gauss-Seidel method, and the SOR method, are employed by many authors. ${ }^{1,7,14}$ For the relaxation method, choosing a proper relaxation factor $\omega$, which determines the convergence rate, is important. The relationship between the relaxation factor and the corresponding iteration times for convergence is shown in Fig. 7. From this relationship we know that the optimal relaxation factor for our algorithm is $\omega_{\text {optimal }}=1.881$ and that the corresponding iteration times for convergence is 111 . 


\section{B. Cholesky Decomposition Method}

Compared with the direct solution algorithm, the iterative method is time consuming. Because of its positive and definite property, the Cholesky decomposition method can be used to solve this equation set. We have $\mathbf{A}^{T} \mathbf{A}>0$, after adding a constraint condition. So there exists at least a lower triangular matrix $\mathbf{B}$ that satisfies $\mathbf{A}^{T} \mathbf{A}=\mathbf{B B}^{T}$. If we restrict each diagonal element of $\mathbf{B}$ to be positive, then the corresponding decomposition $\mathbf{A}^{T} \mathbf{A}=\mathbf{B B}^{T}$ will be unique, where $\mathbf{B}$ is an invertible matrix.

If we assume

$$
\mathbf{A}^{T} \mathbf{A}=\left[a_{i j}\right]_{m \times m}, \quad \mathbf{B}=\left[b_{i j}\right]_{m \times m}=\left\{\begin{array}{ll}
0, & i<j \\
b_{i j}, & i \geq j
\end{array},\right.
$$

then we have

$$
a_{i j}=\sum_{k=1}^{i} b_{i k} b_{j k}, \quad(1 \leq i \leq j \leq m) .
$$

Therefore the elements of the lower triangular matrix $\mathbf{B}$ are obtained. Let the normal equation set be $\mathbf{A}^{T} \mathbf{A W}=\mathbf{Y}$; the steps for solving the equation set employing Cholesky decomposition method are as follows:

1. Decomposition. Make a Cholesky decomposition, $\mathbf{A}^{T} \mathbf{A}=\mathbf{B B}^{T}$; then the normal equations become

$$
\mathbf{B B}^{T} \mathbf{W}=\mathbf{Y} \text {. }
$$

Let

$$
\mathbf{U}=\mathbf{B}^{T} \mathbf{W}, \quad \mathbf{U}=\left(u_{1}, u_{2}, \ldots, u_{m}\right)^{T} .
$$

Therefore

$$
\mathbf{B U}=\mathbf{Y} .
$$

2. Substitution. From Eq. (5.5) we get the forward substitution formula for calculating $\mathbf{U}$ :

$$
\begin{aligned}
& u_{1}=y_{1} / b_{11}, \\
& u_{i}=\left(y_{i}-\sum_{j=1}^{i-1} b_{i j} y_{j}\right) / b_{i i}, \quad i=2,3, \ldots, m .
\end{aligned}
$$

From Eq. (5.4) we get the backward substitution formula for calculating $\mathbf{W}$ :

$$
\begin{aligned}
w_{m} & =u_{m} / b_{m, m}, \\
w_{i} & =\left(u_{i}-\sum_{j=i+1}^{m} b_{i i} w_{j}\right) / b_{i i}, \\
i & =m-1, m-2, \ldots, 1 .
\end{aligned}
$$

One of the features of the generalized reconstruction algorithm introduced in this paper is that the normal coefficient matrix $\mathbf{A}^{T} \mathbf{A}$ is invariable to a large extent, regardless of the complexity of the boundary encountered. In addition, the Cholesky decomposition has nothing to do with the vectors on the right-hand side of the equations, and decomposition would be performed before substitution. Therefore the decompo- sition, once performed, is always suitable in the process of repeated equation solving computations; so a lot of computation time is spared.

\section{Memory Problem in Solving Normal Equations}

As far as the large sparse linear equation set is concerned, the storage of its coefficient matrix is the primary problem and should be solved first. This problem has been mentioned in the literature.1,12 We usually employ the compressed storage method, eliminating the unnecessary memory space for zero elements.

However, in our problem, even if the compressed storage method is used, the storage for the nonzero remainders and pointer vectors still take up too much space. In addition, the Cholesky decomposition also generates a nonzero band matrix $\mathbf{b}(j, i)$ whose bandwidth is $t+1$; so we need at least $m \times(t+1)$ elements of memory space for saving this band matrix.

It is easy to find that, no matter how large this coefficient matrix is, its components always consist of only 1-4 and 0. Therefore we can express the matrix as a function $\mathbf{a}(i, j)$ whose values are the five elements $1-4$ and 0 , where $i$ denotes the row index and $j$ denotes the column index. In this way we do not need a specialized memory space for the coefficient matrix even if the mesh number of the square net is increased. However, this strategy is not applicable to the Gaussian elimination method, in which each matrix element should be assigned a storage space for the purpose of the computations between elements.

\section{Error Analysis}

According to Appendix B the normal equations of Eq. (3.7) at interior points correspond to the cross scheme of partial differential equation (4.10), whose truncation errors are of $a^{4}$ order of magnitude; the truncation errors of the normal equations at boundary points are of $a^{3}$ order of magnitude. Therefore the smaller proportion the normal equations of boundary points occupy, the greater the precision of the normal equation solutions will be. For example, in the integration domain $\boldsymbol{\Omega}_{0}$ before extension, there are a total of 161 normal equations, $\sim 40$ of which correspond to boundary points. This suggests that the ratio of the number of normal equations of $a^{3}$ order of magnitude to that of normal equations of $a^{4}$ order of magnitude is approximately 1:3.

Set

$$
\begin{aligned}
& \mathbf{W}_{161}=\left(w_{1}, w_{2}, \ldots, w_{161}\right)^{T}, \\
& \mathbf{p} \mathbf{W}_{i}^{(3)} \in\left(\left.\frac{\partial^{3} \mathbf{W}}{\partial y^{3}}\right|_{i},\left.\frac{\partial^{3} \mathbf{W}}{\partial z^{3}}\right|_{i}\right), \quad \mathbf{p} \mathbf{W}_{i}^{(4)} \in\left(\left.\frac{\partial^{4} \mathbf{W}}{\partial y^{4}}\right|_{i},\left.\frac{\partial^{4} \mathbf{W}}{\partial z^{4}}\right|_{i}\right), \\
& \left.\left(\frac{\partial^{4}}{\partial y^{4}}+\frac{\partial^{4}}{\partial z^{4}}\right) \mathbf{W}\right|_{i}=M_{i}^{(4)},\left.\left(\frac{\partial^{3}}{\partial y^{3}}+\frac{\partial^{3}}{\partial z^{3}}\right) \mathbf{W}\right|_{i}=M_{i}^{(3)} .
\end{aligned}
$$

After extension, however, the domain of definition of the wave-front function $\mathbf{W}(y, z)$ is enlarged to the 
domain $\boldsymbol{\Omega}_{1}$. If $\overline{\boldsymbol{\Omega}}_{0} \subset \boldsymbol{\Omega}_{1}$ and $\partial \boldsymbol{\Omega}_{1} \cap \boldsymbol{\Omega}_{0}=\Phi$, then the boundary condition $\partial \mathbf{W} /\left.\partial \mathbf{n}\right|_{\partial \Omega_{1}}=0$ is defined and strictly satisfied without introducing errors. For an interior point of $\boldsymbol{\Omega}_{1}$ we eliminate the higher terms in Eq. (A14) and rewrite it as follows:

$$
\begin{aligned}
-w_{i+1}^{\prime}-w_{i+t}^{\prime} & +4 w_{i}^{\prime}-w_{i-1}^{\prime}-w_{i-t}^{\prime} \\
& =-a^{2} \nabla \mathbf{n}_{i}-\frac{\mathbf{a}^{4}}{12}\left(\left.\frac{\partial^{4} \mathbf{W}^{\prime}}{\partial y^{4}}\right|_{i}+\left.\frac{\partial^{4} \mathbf{W}^{\prime}}{\partial \mathbf{z}^{4}}\right|_{i}\right) .
\end{aligned}
$$

We still let $\mathbf{W}_{0}$ denote the ideal wave-front function and $\mathbf{W}$ denote the actual reconstructed wave-front function, and $\mathbf{n}(\mathrm{y}, \mathrm{z})$ denotes the gradient error function; thus we have

$$
\begin{aligned}
\mathbf{W}^{\prime} & =\mathbf{W}-\mathbf{W}_{0}=\left[w_{1}^{\prime}, w_{2}^{\prime}, \ldots, w_{m}^{\prime}\right]^{T}, \\
\mathbf{N} & =\left[\mathbf{n}_{1}, \mathbf{n}_{2}, \ldots, \mathbf{n}_{m}\right]^{T}, \\
\left(\frac{\partial^{4}}{\partial y^{4}}+\frac{\partial^{4}}{\partial z^{4}}\right) \mathbf{W}^{\prime} & =\left[M_{1}^{(4)}, M_{2}^{(4)}, \ldots, M_{m}^{(4)}\right]^{T}=\mathbf{M}_{4},
\end{aligned}
$$

where $\mathbf{n}_{i}=0$ in Eq. (6.4) when $(y, z) \in \boldsymbol{\Omega}_{1} \backslash \boldsymbol{\Omega}_{0}$, and $M_{i}^{(4)}=0$ in Eq. (6.5) when $(y, z) \in \partial \boldsymbol{\Omega}_{1}$. Note that

$$
\mathbf{n}_{i}(y, z)=\mathbf{n}_{y}(y, z) \cdot i+\mathbf{n}_{z}(y, z) \cdot j ;
$$

thus

$$
\nabla \mathbf{n}_{i}=\left.\frac{\partial \mathbf{n}_{y}}{\partial y}\right|_{i}+\left.\frac{\partial \mathbf{n}_{z}}{\partial z}\right|_{i}
$$

Let $\left.n_{y}\right|_{i} ^{i+1}$ express the value of $n_{y}$ at the midpoint between grid point $i$ and grid point $i+1$, and let $\left.n_{z}\right|_{i+t} ^{i}$ express the value of $n_{z}$ at the midpoint between grid point $i$ and grid point $i+t$ (see Fig. 8). According to formulas (A31) and (A32), we have

$$
\begin{aligned}
& \left.\frac{\partial \mathbf{n}_{y}}{\partial y}\right|_{i}=\frac{\left.n_{y}\right|_{i} ^{i+1}-\left.n_{y}\right|_{i-1} ^{i}}{a}-\left.\frac{a^{2}}{4 !} \frac{\partial^{3} \mathbf{n}_{y}}{\partial y^{3}}\right|_{i}+\mathbf{O}\left(a^{4}\right), \\
& \left.\frac{\partial \mathbf{n}_{z}}{\partial z}\right|_{i}=\frac{\left.n_{z}\right|_{i} ^{i-t}-\left.n_{z}\right|_{i+t} ^{i}}{a}-\left.\frac{a^{2}}{4 !} \frac{\partial^{3} \mathbf{n}_{z}}{\partial z^{3}}\right|_{i}+\mathbf{O}\left(a^{4}\right) .
\end{aligned}
$$

when we apply Eqs. (6.7) and (6.8) to formula (6.6), then apply Eq. (6.6) to Eq. (6.2), we have

$$
\begin{aligned}
-w_{i+1}^{\prime}-w_{i+t}^{\prime}+ & 4 w_{i}^{\prime}-w_{i-1}^{\prime}-w_{i-t}^{\prime} \\
=a\left[-\left.n_{y}\right|_{i} ^{i+1}+\right. & \left.\left.n_{y}\right|_{i-1} ^{i}-\left.n_{z}\right|_{i} ^{i-t}+\left.n_{z}\right|_{i+t} ^{i}\right]+\frac{a^{4}}{24}\left[\left.\frac{\partial^{3} \mathbf{n}_{y}}{\partial y^{3}}\right|_{i}\right. \\
& \left.+\left.\frac{\partial^{3} \mathbf{n}_{z}}{\partial z^{3}}\right|_{i}-2\left(\left.\frac{\partial^{4} \mathbf{W}^{\prime}}{\partial y^{4}}\right|_{i}+\left.\frac{\partial^{4} \mathbf{W}^{\prime}}{\partial z^{4}}\right|_{i}\right)\right] .
\end{aligned}
$$

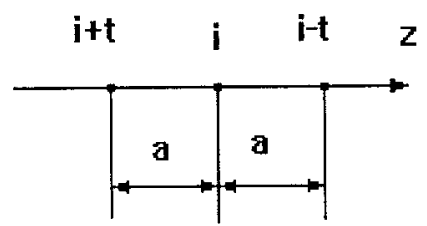

Fig. 8. Situation of adjacent points in one direction.
Let

$$
\left(\frac{\partial^{3}}{\partial y^{3}} i+\frac{\partial^{3}}{\partial z^{3}} j\right) \mathbf{N}=\left[\mathbf{n}_{1}^{(3)}, \mathbf{n}_{2}^{(3)}, \ldots, \mathbf{n}_{m}^{(3)}\right]=\mathbf{M}_{3} .
$$

When $(y, z) \in \boldsymbol{\Omega}_{1} \backslash \boldsymbol{\Omega}_{0}$, all terms on the right-hand side of Eq. (6.9) are zero. For convenience of expression we still express the noise vectors $\left(\left.n_{y}\right|_{i},\left.n_{z}\right|_{i}\right)$ as a $2(m-$ $t) \times 1$ matrix $\mathbf{N}^{\prime}$, in which the elements $\left.n_{y}\right|_{i}$ and $\left.n_{z}\right|_{i}$ are zero when they belong to $\boldsymbol{\Omega}_{1} \backslash \boldsymbol{\Omega}_{0}$.

Therefore, comparing Eq. (6.9) with Eq. (3.18), we have

$$
\mathbf{A}^{T} \mathbf{A} \mathbf{W}^{\prime}=\frac{a^{4}}{24}\left(\mathbf{M}_{3}-2 \mathbf{M}_{4}\right)+a \mathbf{A}^{T} \mathbf{N}^{\prime},
$$

where matrix $\mathbf{A}$ is the same matrix of $\mathbf{A}$ in Eq. (3.5). This is an important equation, which defines the relationship between wave-front estimation errors and the discretization errors of the algorithm together with the measurement errors of wave-front gradients.

We introduce norm $\|\cdot\|_{2}$

$$
\|\mathbf{X}\|_{2}=\left(\mathbf{X}^{T} \mathbf{X}\right)^{1 / 2}=\left(\sum_{i=1}^{m}\left|x_{i}\right|^{2}\right)^{1 / 2}
$$

and the corresponding matrix norm

$$
\operatorname{lub}_{2}(\mathbf{A})=\max _{x \neq 0}\left(\frac{\mathbf{X}^{T} \mathbf{A}^{T} \mathbf{A} \mathbf{X}}{\mathbf{X}^{T} \mathbf{X}}\right)^{1 / 2}=\left[\rho\left(\mathbf{A}^{T} \mathbf{A}\right)\right]^{1 / 2},
$$

where $\rho\left(\mathbf{A}^{T} \mathbf{A}\right)$ is the spectral radius of $\mathbf{A}^{T} \mathbf{A}$. Therefore we have (see Appendix D)

$$
\begin{aligned}
\left\|\mathbf{W}^{\prime}\right\|_{2} \leq & \frac{a^{4}}{24} \frac{\operatorname{cond}\left(\mathbf{A}^{T} \mathbf{A}\right)}{\operatorname{lub}_{2}(\mathbf{A})^{2}}\left\|\mathbf{M}_{3}-2 \mathbf{M}_{4}\right\|_{2} \\
& +a \frac{\left[\operatorname{cond}\left(\mathbf{A}^{T} \mathbf{A}\right)\right]^{1 / 2}}{\operatorname{lub}_{2}(\mathbf{A})}\left\|\mathbf{N}^{\prime}\right\|_{2} .
\end{aligned}
$$

We assume that there are $k$ measurement points in $\boldsymbol{\Omega}_{0}$ and that the distributions of all the measurement errors of wave-front partial derivatives are independent. Let $\sigma_{G_{y}}$ denote the rms value of the partial derivative error distribution in the $y$ direction, and let $\sigma_{G_{z}}$ denote the rms value of the partial derivative error distribution in the $z$ direction. Set

$$
\begin{array}{r}
\mathbf{N}^{\prime}=\left[0, \ldots,\left.n_{N_{y}}\right|_{1},\left.n_{N_{y}}\right|_{2}, \ldots,\left.n_{N_{y}}\right|_{k}, 0, \ldots, 0, \ldots,\right. \\
\left.\left.n_{N_{z}}\right|_{1},\left.n_{N_{z}}\right|_{2}, \ldots,\left.n_{N_{z}}\right|_{k}, 0, \ldots\right]_{2(m-t)}^{T},
\end{array}
$$

where

$$
\left.n_{N_{y}}\right|_{i}=\left.n_{y}\right|_{j} ^{j+1},\left.n_{N_{z}}\right|_{i}=\left.n_{z}\right|_{j+t} ^{j}, i=1,2, \ldots, k .
$$

Therefore

$$
\left\|\mathbf{N}^{\prime}\right\|_{2}=\left[\sum_{i=1}^{k}\left(\left[\left.n_{N_{y}}\right|_{i}\right]^{2}+\left[\left.n_{N_{z}}\right|_{i}\right]^{2}\right)\right]^{1 / 2}=\sqrt{k}\left({\sigma_{G_{y}}}^{2}+{\sigma_{G_{z}}}^{2}\right)^{1 / 2},
$$


where

$$
\sigma_{G_{y}}=\left(\frac{1}{k} \sum_{i=1}^{k}\left[n_{N_{y}} \mid i\right]^{2}\right)^{1 / 2}, \quad \sigma_{G_{z}}=\left(\frac{1}{k} \sum_{i=1}^{k}\left[n_{N_{z}} \mid i\right]^{2}\right)^{1 / 2} .
$$

If the reducing system is introduced, and if we let $\beta$ represent the reciprocal of the magnifying power of the reducing system, from formula (2.2), we know that $\sigma_{G_{y}}, \sigma_{G_{z}}, \sigma_{\mathrm{CCD}_{y}}$, and $\sigma_{\mathrm{CCD}_{z}}$ have the following relationships:

$$
\sigma_{G_{y}}=\frac{\beta \sigma_{\mathrm{CCD}_{y}}}{f_{0}}, \quad \sigma_{G_{z}}=\frac{\beta \sigma_{\mathrm{CCD}_{z}}}{f_{0}},
$$

where $f_{0}$ is the focal length of the lenslet array. In addition,

$$
\left\|\mathbf{W}^{\prime}\right\|_{2}=\sqrt{m} \sigma_{w^{\prime}}=t \sigma_{\mathbf{W}^{\prime}} .
$$

Therefore, according to relation (6.14), we have

$$
\begin{aligned}
\sigma_{\mathbf{W}^{\prime}} \leq & \frac{a^{4}}{24 t} \frac{\operatorname{cond}\left(\mathbf{A}^{T} \mathbf{A}\right)}{\operatorname{lub}_{2}(\mathbf{A})^{2}}\left\|\mathbf{M}_{3}-2 \mathbf{M}_{4}\right\|_{2} \\
& +\frac{a \beta \sqrt{k}}{f_{0} t} \frac{\left[\operatorname{cond}\left(\mathbf{A}^{T} \mathbf{A}\right)\right]^{1 / 2}}{\operatorname{lub}_{2}(\mathbf{A})} \sigma_{\mathrm{CCD}},
\end{aligned}
$$

where $\sigma_{\mathrm{CCD}}=\left(\sigma_{\mathrm{CCD}_{\gamma}}{ }^{2}+\sigma_{\mathrm{CCD}_{z}}{ }^{2}\right)^{1 / 2}$ is the measurement rms error of CCD, whose unit is the same as that of the wave-front rms error $\sigma_{w^{\prime}}$. This formulation gives an error estimation of the reconstructed wave front resulting from the discretization errors of the algorithm and the measurement errors of wave-front gradients.

Employing numerical calculation, we get $\operatorname{lub}_{2}(\mathbf{A})=$ 2.82 when $t=17$ and $\operatorname{lub}_{2}(\mathbf{A})=2.81$ when $t=15$; so the difference between the two values can be neglected. However, $\operatorname{lub}_{2}\left[\left(\mathbf{A}^{T} \mathbf{A}\right)^{-1}\right]$ changes greatly when $t$ and the boundary constraints change; correspondingly, the condition number of the normal equations varies significantly. Figure 9 gives the relationships of constraint points and the corresponding condition numbers of the normal equations. It shows that, when $t=17$ together with the constraint point $w_{145}=0.0$, the condition number of the corresponding normal equations reaches its minimum value $146.8 \times 1.93=1161.5$; when $t=15$ together with the constraint point $w_{123}=0.0$, the corresponding condition number of the corresponding normal equations reaches its minimum value $109.896 \times$ $7.9126=869.35$. Therefore, applying $t=15$ and $k=161$ in formula $(6.20)$, we have

$$
\sigma_{\mathbf{W}^{\prime}} \leq 0.305 a^{4}\left\|\mathbf{M}_{3}-2 \mathbf{M}_{4}\right\|_{2}+8.876 \frac{a \beta}{f_{0}} \sigma_{\mathrm{CCD}} .
$$

Generally, $\left\|\mathbf{M}_{3}-2 \mathbf{M}_{4}\right\|_{2}$ is a small value of $a^{4}$ order of magnitude. The first term of the right-hand side of this inequality is a discretization error concerned with the algorithm we adapted, which has a higher small value approaching zero quickly at the rate of $a^{4}$. So the first term can be neglected compared with the
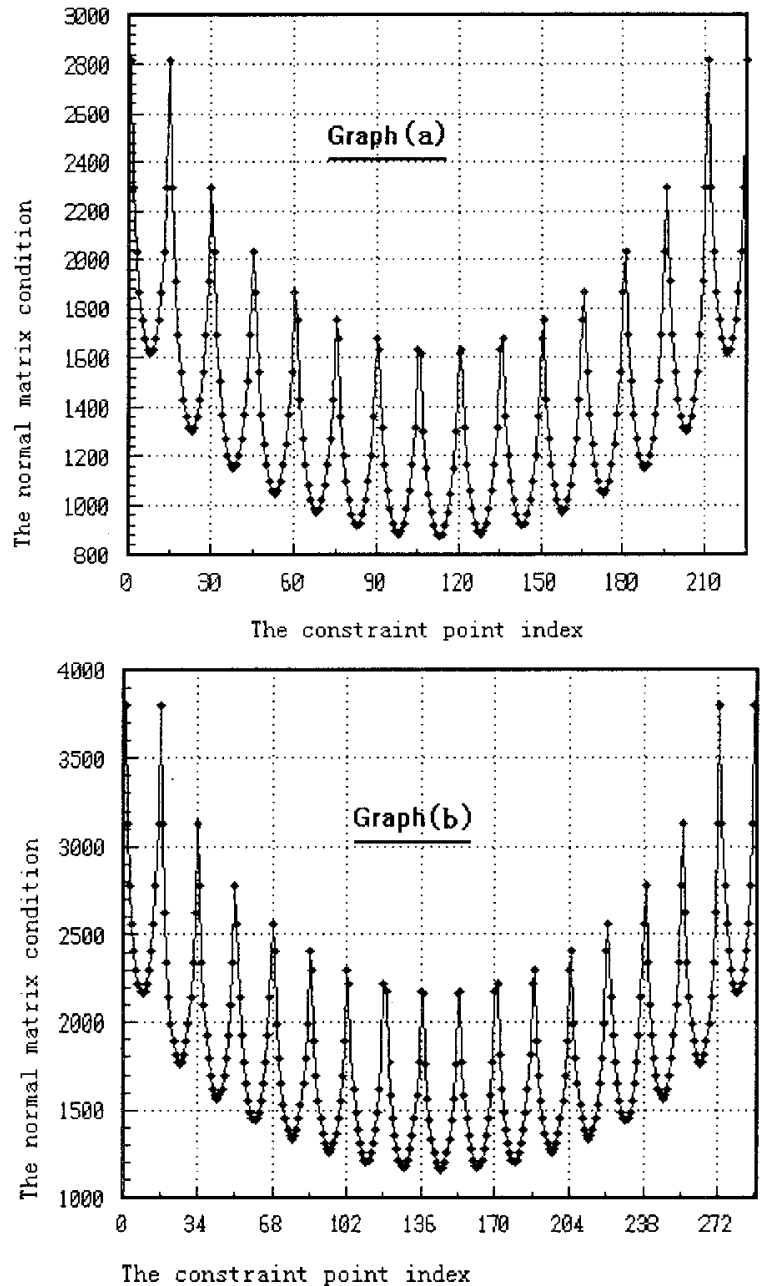

Fig. 9. Relationship between the constraint points (zero point of the reconstructed wave front) and the corresponding condition numbers of the normal equation set. Graph (a) is the case $t=15$, and graph (b) is the case $t=17$. The graphs show that, with the same constraint situation, the condition number of the normal equation set in the case of $t=15$ is better than in the case of $t=$ 17.

second term. Therefore the wave-front rms error depends mainly on the measurement rms error of CCD.

Generally speaking, $a \ll f_{0}$. For example, in the $\mathrm{S}-\mathrm{H}$ system of the first experimental system of thinmirror active optics of NAIRC, $a=2 \mathrm{~mm}, f_{0}=184$ $\mathrm{mm}$, and $\beta=7.5$. Therefore

$$
\sigma_{\mathbf{W}^{\prime}} \leq 17.752\left(\beta / f_{0}\right) \sigma_{\mathrm{CCD}}=0.724 \sigma_{\mathrm{CCD}} .
$$

In conclusion, although the condition number of normal equations is not small, the rms wave-front error is just comparable with the rms measurement error of CCD and even smaller. Moreover, the condition number of normal equations in case of $t=15$ is better than that of $t=17$; this implies that it would be workable if we were to extend the sampling domain to the configuration of $t=15$.

Inequality (6.20) shows that the rms wave-front error $\sigma_{\mathbf{w}^{\prime}}$ is directly related to the $\mathrm{rms}$ measurement error of $\mathrm{CCD}\left(\sigma_{\mathrm{CCD}}\right)$, the mesh size $a$, the focal length of 
lenslet array $f_{0}$, and the magnifying power of the reducing system $\beta$. The condition number of the normal equations, cond $\left(\mathbf{A}^{T} \mathbf{A}\right)$, takes the role of magnifier. It is feasible to adopt some measures to improve the condition number possibly, such as choosing the proper constraints, with the leastsquares minimum-norm solutions, ${ }^{8}$ etc. However, the solutions to the wave-front figure $\mathbf{W}$ that satisfies wave-front gradients are a group of parallel surfaces, so the added constraint conditions should not break the parallelism of the solutions; otherwise they are not the solutions to this problem. According to the above analysis, we know that the errors generated from the algorithm occupy only a small fraction in the error budget. Approximately, the discretization errors are in direct proportion to the fourth power of mesh size $a$ and sharply approach zero when $a$ decreases. Therefore, to improve the precision of the $\mathrm{S}-\mathrm{H}$ test, we should focus on making the $\mathrm{S}-\mathrm{H}$ grid array denser, developing a longer focal length lenslet array, and improving the CCD measurement precision.

\section{Example and Analysis}

\section{A. Demonstration of Algorithm and Programs}

Take the $\mathrm{S}-\mathrm{H}$ test in the thin-mirror active optics experiment system at NAIRC as an example. Programs are composed in $\mathrm{C}++$ language in terms of the domain extension algorithm. The following two steps are employed to demonstrate the programs and the algorithm.

\section{Checking Computations of the Programs and the Algorithm}

We introduce the function

$$
\mathbf{f}(z, y)=z^{2}+y^{2} .
$$

Performing derivation calculus on both sides, we have $\partial \mathbf{f} / \partial z=2 z$ and $\partial \mathbf{f} / \partial y=2 y$. Setting the coordinate origin at the geometry center of the extended domain, and letting $t=15$, mesh size $a=0.1$, we have

$$
\left\{\begin{array}{l}
\partial \mathbf{f} /\left.\partial z\right|_{i, j}=2 a i \\
\partial \mathbf{f} /\left.\partial y\right|_{i, j}=2 a j
\end{array}, \quad i, j=0, \pm 1, \pm 2, \ldots, \pm 7 .\right.
$$

Let the numerical values generated by formula (7.2) be initial values in the test area, and let all the initial values in the extended areas be zero. We set the zero point of the wave-front surface at $\mathbf{f}(0,0)=0.0$ and employ the Cholesky method to solve Eq. (7.2). We subtract the solutions obtained from the original numerical values generated by formula (7.1). The residuals are all close to zero as shown in Fig. 10, except for some boundary points. Besides the Cholesky method, the iterative method is employed for cross checking. The two methods agree with each other exactly.

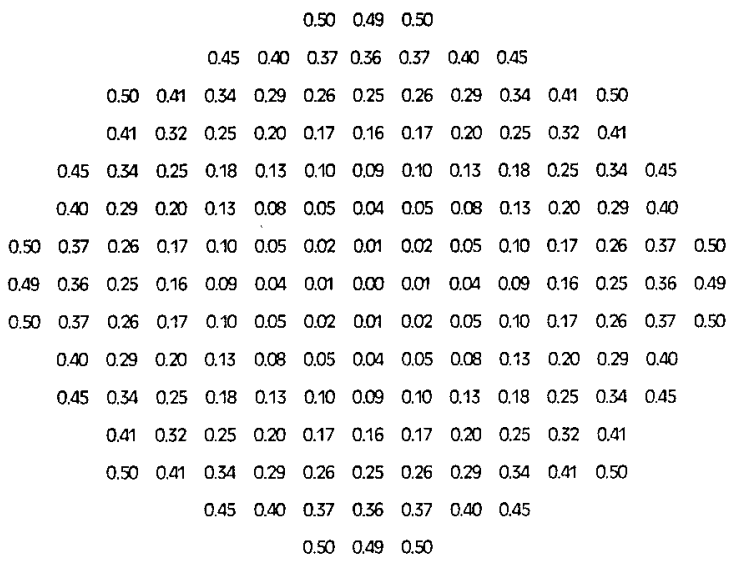

(a)

Num. of S-H grid array: 161

Integration domain: Extended to $15 \times 15$ grid array

Solution method: Cholesky method or Iterative method

$\mathbf{a}=0.1$, Zero point of wave front: $\mathbf{w}_{113}=0.0$,

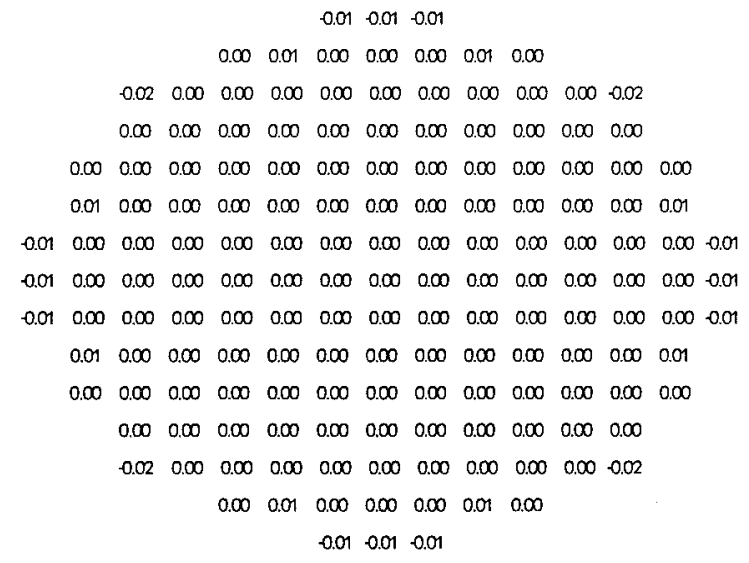

(b)

Fig. 10. (a) is the scalar phase array of an ideal wave front generated by $\mathbf{f}(z, y)=z^{2}+y^{2}$. (b) is the array of phase difference between the ideal wave front and the calculated wave front determined from the gradients of function $\mathbf{f}(z, y)$, with employment of the extension algorithm proposed in this paper.

\section{Stability Demonstration of the Domain Extension Algorithm}

First, the partial derivatives in Eq. (7.2) are assigned as a group of Gaussian distribution random numbers. That is,

$$
\left\{\begin{array}{l}
\partial \mathbf{f} /\left.\partial z\right|_{i, j}=\mathbf{R}_{z}(i, j) \\
\partial \mathbf{f} /\left.\partial y\right|_{i, j}=\mathbf{R}_{y}(i, j)
\end{array}, \quad i, j=0, \pm 1, \pm 2, \ldots, \pm 7,\right.
$$

where $\mathbf{R}_{z}(i, j)$ are Gaussian distributed random errors, which are assigned to zero outside the test domain, the average value of which is 0.0 in the test area and the rms of which is $0.118 . \quad \mathbf{R}_{y}(i, j)$ are Gaussian distributed random errors, which are as- 
signed zero outside the test area, the average value of which is 0.0 in the test area and the rms of which is 0.108. Therefore

$$
\sigma_{\text {error }}=\left(\sigma_{z}^{2}+\sigma_{y}^{2}\right)^{1 / 2}=0.160 .
$$

According to formulas (6.21) and (7.4), omitting the discretization error, we have

$$
\sigma_{\mathbf{W}^{\prime}} / \sigma_{\text {error }} \leq 8.876 a=17.752 .
$$

The Cholesky method and the iterative method are used separately to solve the normal equations, and their results are identical. The data are shown in Fig. 11(a), and Fig. 11(b) shows the corresponding three-dimension surface map of the reconstructed wave front, $\sigma_{\mathbf{W}^{\prime}}=0.189$; so $\sigma_{\mathbf{W}^{\prime}} / \sigma_{\text {error }}=1.18$. This formula is congruous with the error-bound estimation given by formula (7.5) and confirms Fried's formula (35) in the literature. ${ }^{4}$

To make a comparison with the result of the preextension case, we still use the random errors in formula (7.3) as the partial derivative values. The data of the calculation result are shown in Fig. 12(a), and Fig. 12(b) shows the corresponding three-dimension surface map of the reconstructed wave front. The $\mathrm{rms}$ of the wave front is $\sigma_{\mathbf{W}^{\prime}}=0.210$; therefore $\sigma_{\mathbf{W}^{\prime}} /$ $\sigma_{\text {error }}=1.32$. Obviously, it is also congruous with formula (7.5). Of course, the result of the preextension case is more precise, but the domain extension algorithm is better in the control of error propagation.

\section{Analysis on the Effect of Domain Extension}

Comparing Fig. 11 with Fig. 12, we find that there are slight discrepancies. These discrepancies are caused by domain extension and have nothing to do with algorithms employed in the solution of normal equations.

We know that, in the least-squares sense, the wave-front phase of each grid point is the average of wave-front phases of adjacent points plus the average of gradient integrals of adjacent points. However, the integration conditions of the interior points and the boundary points are different. The interior points have four adjacent points and four integration paths, but the boundary points have three (or two) adjacent points and three (or two) integration paths. Therefore the smoothing effect at boundary points is weaker than at interior points, as are the precision and the reliability.

However, after domain extension, the gradients outside the test domains are created (assigned to zero), and the boundary points are changed into interior points. So the adjacent points of the original boundary points consist of real points and virtual points, and the virtual points can be shared by two original boundary points that are not adjacent. This makes the nonadjacent points smooth together by means of virtual adjacent points. Accordingly, the integrations in the original boundary points are changed from three- (or two-) point averaging and three- (or two-) path integral to four-point averaging and fourpath integral. Therefore the real gradients in the
Num. of S-H grid arry:161 Integration domain: Extended Solution method: Cholesky or Iterative method

Zero point of wave front : At $\mathbf{w}_{113}=0.0, \mathrm{t}=15, \mathrm{a}=2$

Avg. of gradient errors:0.046 RMS of gradient errors:0.16 Avg. of wave-front errors:0.017 RMS of wave-front errors:0.19

$0.14 \quad-0.02-0.08$

$\begin{array}{lllllllllllllll}0.09 & 0.01 & -0.02 & -0.03 & -0.05 & -0.18 & 0.17\end{array}$

$\begin{array}{lllllllllll}0.09 & 0.13 & 0.10 & 0.07 & 0.06 & 0.10 & 0.01 & 0.15 & 0.14 & 0.10 & 0.11\end{array}$

$\begin{array}{lllllllllll}0.15 & 0.13 & 0.16 & 0.07 & 0.06 & 0.13 & -0.05 & 0.12 & -0.16 & -0.10 & -0.02\end{array}$

$\begin{array}{lllllllllllll}0.16 & 0.20 & 0.23 & 0.29 & 0.24 & 0.13 & 0.08 & 0.10 & 0.02 & -0.04 & -0.06 & 0.17 & -0.22\end{array}$

$\begin{array}{lllllllllllll}0.09 & 0.13 & 0.26 & 0.32 & 0.24 & 0.27 & 0.19 & -0.02 & 0.02 & -0.03 & -0.12 & -0.26 & -0.30\end{array}$

$\begin{array}{lllllllllllllll}0.01 & 0.11 & 0.12 & 0.13 & 0.23 & 0.32 & 0.27 & 0.15 & 0.01 & -0.03 & 0.01 & 0.06 & 0.14 & 0.31 & 0.24\end{array}$

$\begin{array}{lllllllllllllll}0.20 & 0.27 & 0.22 & 0.21 & 0.26 & 0.22 & 0.16 & 0.00 & -0.02 & 0.02 & 0.11 & 0.07 & 0.19 & 0.23 & 0.40\end{array}$

$\begin{array}{lllllllllllllll}0.22 & 0.37 & 0.31 & 0.31 & 0.23 & 0.16 & 0.17 & 0.07 & 0.00 & -0.09 & 0.17 & -0.21 & 0.24 & -0.30 & 0.36\end{array}$

$\begin{array}{lllllllllllll}0.25 & 0.26 & 0.25 & 0.24 & 0.15 & 0.11 & 0.15 & 0.04 & -0.23 & -0.31 & -0.35 & 0.25 & -0.23\end{array}$

$\begin{array}{lllllllllllll}0.01 & 0.13 & 0.27 & 0.21 & 0.16 & 0.20 & 0.14 & 0.05 & 0.31 & 0.22 & -0.29 & -0.34 & 0.22\end{array}$

$\begin{array}{lllllllllll}0.14 & 0.37 & 0.37 & 0.24 & 0.10 & 0.03 & 0.00 & 0.13 & -0.20 & -0.16 & 0.19\end{array}$

$\begin{array}{lllllllllll}0.20 & 0.26 & 0.32 & 0.28 & 0.11 & -0.10 & -0.11 & -0.09 & -0.19 & -0.13 & -0.13\end{array}$

$\begin{array}{llllllllllllll}0.22 & 0.06 & -0.03 & -0.11 & -0.17 & -0.23 & -0.27\end{array}$

$\begin{array}{llll}-0.03 & -0.16 & -0.33\end{array}$

(a)

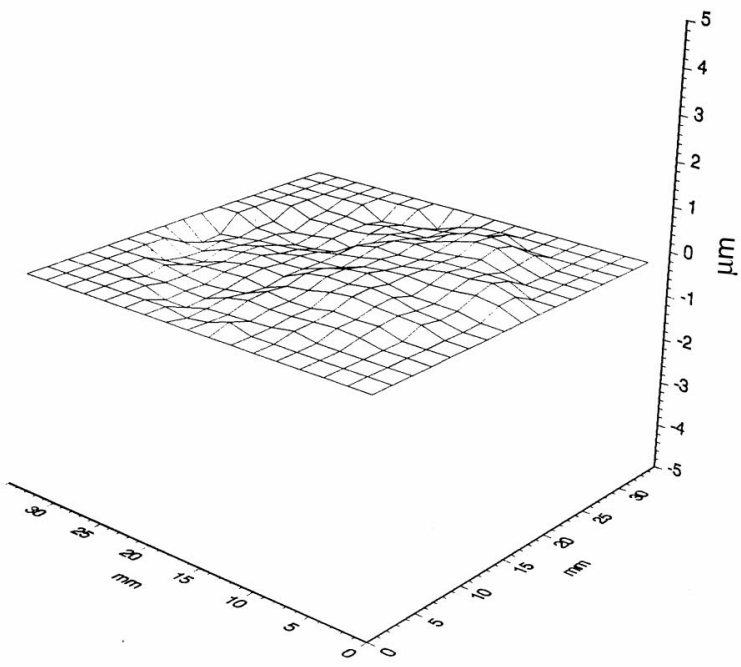

(b)

Fig. 11. Stability demonstration of domain extension algorithm. Using a group of random gradient errors with Gaussian distributions as the initial values and employing the domain extension algorithm, we obtained the reconstructed wave-front phase array shown in (a), and the corresponding three-dimensional map is shown in (b).

boundary are averaged with the virtual gradients (zeros), and that makes the brim of reconstructed wave front a little drooped (see Figs. 13 and 14). For a large aberration system this small droop can be ignored in active optics and adaptive optics as well as in the figure test of mirror polishing; for a small aberration system this droop effect becomes weaker and can thus be neglected. Therefore the domain extension algorithm is reasonable for most practical application cases. 
Num. of S-H grid array: 161 Integration domain: Not extended Solution method: Iterative method

Zero point of wave front : At $\mathbf{w}_{113}=0.0, \mathbf{t}=15, \quad \mathrm{a}=2$

Avg. of gradient errors:0.046 RMS of gradient errors:0.16

Avg. of wave-front errors:0.016 RMS of wave-front errors:0.21

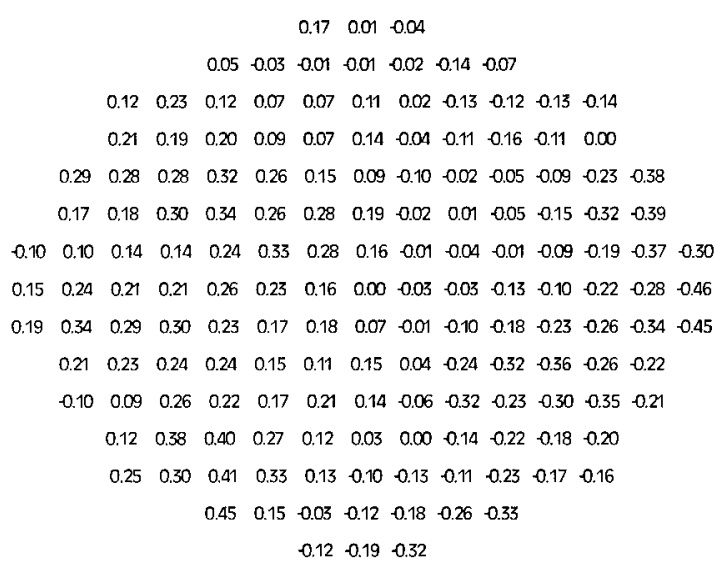

(a)

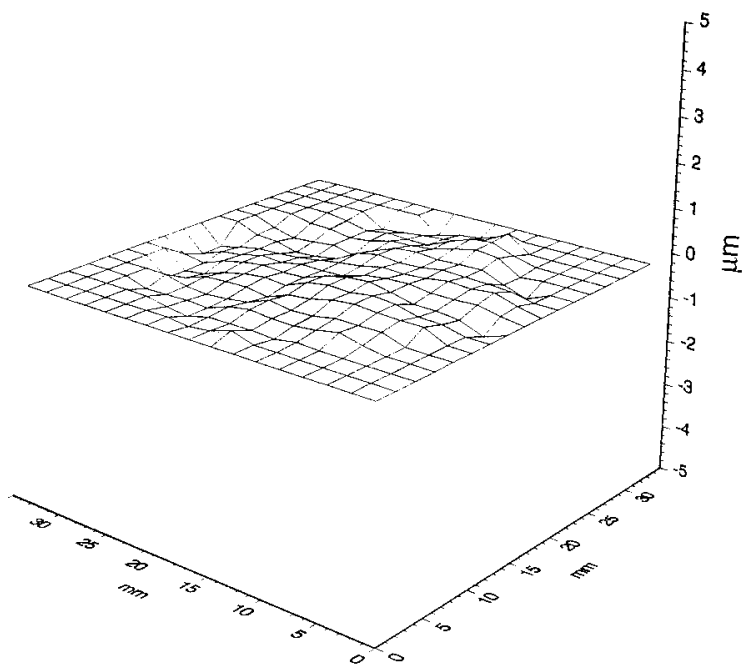

(b)

Fig. 12. Stability demonstration of nonextension algorithm. Using the same group of random gradient errors as in Fig. 11 as the initial values and employing the nonextension algorithm, we obtained the reconstructed wave-front phase array shown in (a), and the corresponding three-dimensional map is shown in (b).

\section{B. Example}

A group of CCD data obtained in the experiment of active optics is used to demonstrate the algorithm proposed. We employ the Cholesky method as well as the iterative method to solve the normal equations. Figure 13 shows the result of the calculation with use of the extension algorithm. For comparison, the result of the calculation with use of the nonextension algorithm is shown in Fig. 14 .
Num. of S-H grid array: 161 Integration domain: Extended Solving method: Cholesky method or Iterative method

Zero point of wave front: At $\mathbf{w}_{113}=0.0, \quad t=15, \mathrm{a}=2$ Avg. of wave-front values:-0.49 RMS of wave-front values:0.36

$$
\begin{array}{rr}
-1.31 & -1.07 \quad 0.90
\end{array}
$$

$\begin{array}{lllllllllllllllll}-1.19 & 1.14 & 0.90 & 0.63 & 0.51 & 0.54 & 0.66\end{array}$

$\begin{array}{llllllllllll}0.74 & 0.97 & 0.99 & 0.79 & 0.54 & 0.31 & 0.13 & 0.15 & 0.47 & 0.86 & 0.96\end{array}$

$\begin{array}{lllllllllllllllll}0.58 & -0.70 & -0.73 & 0.67 & 0.46 & -0.14 & 0.05 & -0.16 & -0.50 & 0.75 & 0.75\end{array}$

$\begin{array}{lllllllllllll}0.47 & -0.55 & 0.71 & 0.84 & 0.66 & 0.31 & 0.10 & -0.09 & 0.23 & -0.47 & 0.53 & 0.55 & 0.63\end{array}$

$\begin{array}{lllllllllllll}0.44 & 0.55 & 0.81 & 0.78 & 0.47 & 0.24 & 0.13 & 0.02 & 0.19 & 0.35 & 0.30 & 0.37 & 0.47\end{array}$

$\begin{array}{lllllllllllllll}-0.37 & 0.43 & 0.69 & 0.82 & 0.57 & 0.35 & 0.17 & 0.04 & 0.02 & 0.05 & 0.07 & 0.10 & -0.21 & -0.35 & 0.51\end{array}$

$\begin{array}{lllllllllllllll}0.37 & 0.46 & 0.72 & -0.77 & 0.49 & 0.31 & 0.15 & 0.00 & 0.09 & 0.09 & 0.08 & 0.02 & 0.13 & -0.28 & -0.44\end{array}$

$\begin{array}{lllllllllllllll}0.47 & 0.52 & -0.72 & 0.77 & 0.52 & -0.33 & 0.17 & 0.03 & 0.08 & 0.13 & 0.12 & 0.05 & 0.11 & 0.29 & 0.45\end{array}$

$\begin{array}{lllllllllllll}0.58 & 0.64 & 0.71 & 0.66 & 0.44 & 0.26 & 0.09 & 0.03 & 0.09 & 0.09 & 0.00 & 0.16 & 0.36\end{array}$

$\begin{array}{lllllllllllll}0.68 & 0.67 & 0.71 & 0.80 & 0.67 & 0.43 & -0.22 & 0.07 & 0.00 & 0.02 & 0.11 & 0.26 & -0.44\end{array}$

$\begin{array}{lllllllllll}0.77 & 0.86 & 0.98 & 0.92 & 0.67 & 0.44 & 0.25 & 0.17 & 0.19 & 0.26 & 0.40\end{array}$

$\begin{array}{llllllllllllllll}0.96 & -1.07 & -1.08 & -1.14 & -1.03 & -0.74 & 0.50 & -0.40 & -0.39 & -0.46 & 0.53\end{array}$

$\begin{array}{lllllllllll}-1.30 & -1.43 & -1.30 & -0.99 & 0.76 & -0.65 & -0.62\end{array}$

$\begin{array}{llll}-1.48 & -1.23 & 0.99\end{array}$

(a)

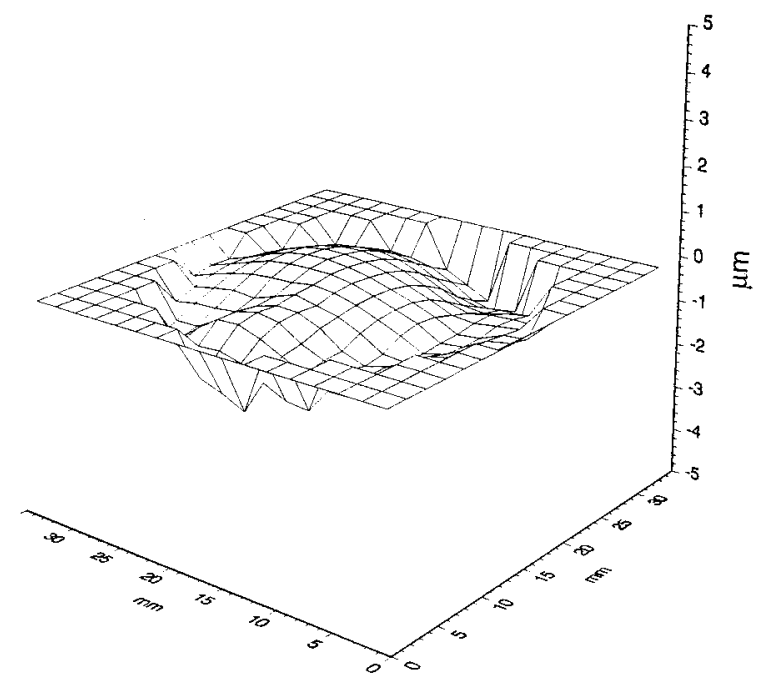

(b)

Fig. 13. Result of calculation with the domain extension algorithm. The reconstructed wave-front phase array is shown in (a). The calculated phase values in the extended domain are discarded, because they are not of interest.

\section{Summary and Generalization}

\section{A. Summary}

The wave-front reconstruction technique is an important branch of modern optical test, and the ShackHartmann $(\mathrm{S}-\mathrm{H})$ test is a new method in optical test that has attracted great interest recently. Based on the studies of $\mathrm{Su}$ et al. ${ }^{14,15}$ a generalized wave-front reconstruction algorithm has been proposed. That is, by extension of the wave-front integration domains to a square net, a generalized normal equation set for wave-front reconstruction is obtained. 
Num. of \$-H grid arry:161 Integration domain: Not extended Solving method: Iterative method

Zero point of wave front: At $\mathbf{W}_{81}=0.0, \mathrm{t}=15, \mathrm{a}=2$

Avg. of wave-front values:-0.49 RMS of wave-front values:0.38

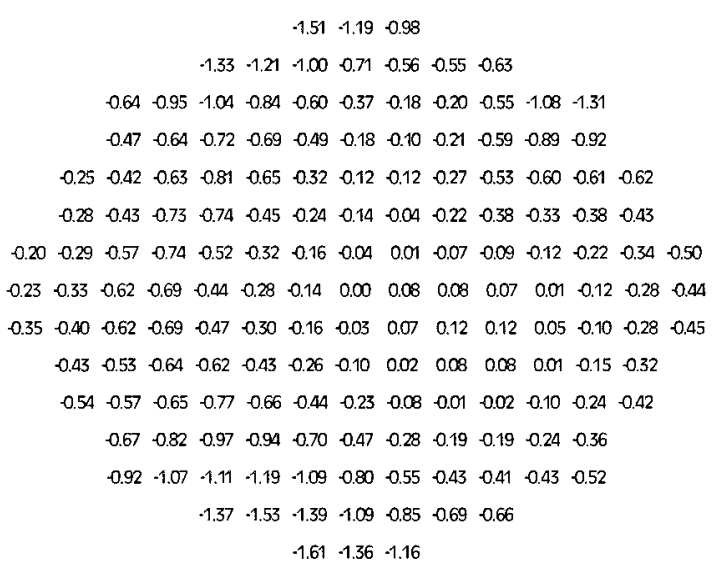

(a)

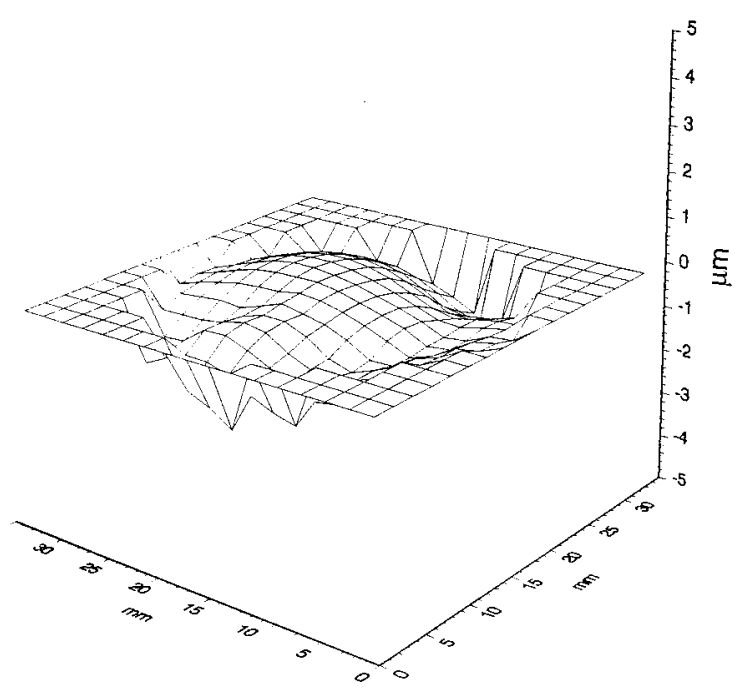

(b)

Fig. 14. Result of calculation with the nonextension algorithm. The reconstructed wave-front phase array is shown in (a), and the corresponding three-dimensional map is shown in (b).

Deeper investigation reveals that the essence of the gradient-based wave-front reconstruction is the Neumann boundary-value problem of the Poission equation in mathematics. That implies that we can apply some algorithms originally developed for difference differential equations to solving the wavefront reconstruction problem. At first, the author tried to prove the compatibility of the domain extension by using the Green's formula. However, because the gradients were assigned to zero outside the former domain during extension, the wave-front function around the boundary of the former domain does not satisfy the second-order differentiable condition. Thus the compatibility of the domain extension is not precisely satisfied. However, we know that the optical mirror surface is usually exceptionally smooth and that its gradient variances (especially in boundary areas) are relatively small. Furthermore, to get a higher precision, the measurement errors need to be smoothed away in the wavefront reconstruction. In view of these reasons the domain extension method is rather reasonable. The given example shows that the reconstructed wave front employing the extension method is just slightly different from that of the traditional method.

Nowadays, not only do many large telescopes use the $\mathrm{S}-\mathrm{H}$ test in their active optics systems and adaptive optics systems, but the test has also become one of the important methods in optical shop testing. ${ }^{18}$ Thus a question is proposed: What attitude should we take toward the algorithm and the precision of wave-front reconstruction? The current study supports that the reconstruction algorithm would be operable if the precision due to the algorithm reached a sufficiently high level. The error analysis shows that the wave-front errors due to the algorithm are much less than those due to CCD measurement errors in the domain extension algorithm. Therefore the accuracy of four-path integration (adopted in this paper) is high enough in actual wave-front reconstruction, and the precision of the algorithm with more than four integration paths ${ }^{11}$ would not be improved significantly, if it were improved. To improve the precision of the wave-front reconstruction, we should try to make the $\mathrm{S}-\mathrm{H}$ grid array denser (reducing the mesh size), to make the focal length of lenslets longer, and to improve the precision of the CCD measurement.

Citing the result data of thin-mirror active optics in China, ${ }^{15}$ we can obtain the CCD position measurement accuracy as $0.162 \mu \mathrm{m} \mathrm{rms} \mathrm{(according} \mathrm{to} \mathrm{a} \mathrm{recent}$ development, the CCD measurement accuracy achieved can be much better than this value). For algorithms without domain extension the wave-front rms error induced by the CCD measurement errors is $0.162 \times 1.32 \times 7.5 / 184=8.7 \times 10^{-3} \mu \mathrm{m}$, which is $\sim 1 / 57 \lambda$; for the algorithm with domain extension the wave-front rms error is $\sim 1 / 64 \lambda$.

The memory-storage problem is the main problem encountered in solving a large linear equation set. Because of the extreme uniformity and regularity of the normal equation set after domain extension, this problem is successfully solved when we express the coefficients of a normal equation set as a function of indexes of the columns and the rows. ${ }^{19}$ With the memory-problem solved, an efficient direct algorithm in solving the equations, the Cholesky method, can be employed successfully to solve the equation set. This makes this domain extension algorithm of wave-front reconstruction quick and efficient. 
B. Generalizing this Extension Algorithm to a Fast Focal Ratio Optical System

The discussion of the generalized extension algorithm proposed in this paper is based on compositum; so it is suitable for different irregular pupil cases. If the focal ratio of the optical system to be measured is faster than that of $\mathrm{S}-\mathrm{H}$ test apparatus, we need to rotate the $\mathrm{S}-\mathrm{H}$ apparatus along the focus 2 of the optical system to be tested (as shown in Fig. 1), thereby satisfying that the movement value of the exit pupil of the optical system to be measured is integer times mesh size $a$ in the $y$ or the $z$ direction relative to the focal plane of lenslet array. Therefore the entrance pupil of the $\mathrm{S}-\mathrm{H}$ test will cover the whole pupil of the optical system to be measured alternately then arrange the indices of $l_{y i}$ and $l_{z i}$ obtained at each time according to the serial number of $\boldsymbol{\Omega}_{1}$. We can arrive at the solution by bringing $l_{y i}$ and $l_{z i}$ to the generalized normal equations. Similarly, if the mesh size is too large to satisfy the precision required, we can rotate the $\mathrm{S}-\mathrm{H}$ apparatus in the meridian plane ( $y$ axis included) and in the azimuthal plane ( $z$ axis included) separately, thus satisfying that the movement value of the pupil image of the optical system to be measured is $a / 2$ or $a / 4$, etc. Therefore new measurement points are inserted into the original measurement points, and the precision requirement will be satisfied. This method avoids the complicated computations by employment of the object functions. ${ }^{20}$ It is another wave-front reconstruction technique for subaperture testing and connections.

Appendix A: Demonstration of $\operatorname{Rank}(A)=m-1$

The matrices in Appendix A were formulated by Zhang. ${ }^{21}$

Set

$$
\mathbf{A}_{0}=\left[\begin{array}{cccc}
-1 & 1 & & \\
& \ddots & \ddots & \\
& & -1 & 1
\end{array}\right]_{(t-1) \times t}
$$

Obviously $\operatorname{rank}\left(\mathbf{A}_{0}\right)=t-1 ; \quad$ if $\mathbf{I}$ is a unit matrix, we have
Adding all the block matrices in columns to the first column, we have

$$
\mathbf{A} \sim\left[\begin{array}{ccccc}
\mathbf{A}_{0} & & & & \\
\mathbf{A}_{0} & \mathbf{A}_{0} & & & \\
\vdots & & \ddots & & \\
\mathbf{A}_{0} & & & \mathbf{A}_{0} & \\
\mathbf{A}_{0} & & & & \mathbf{A}_{0} \\
0 & -\mathbf{I} & & & \\
0 & \mathbf{I} & -\mathbf{I} & & \\
\vdots & & \ddots & \ddots & \\
0 & & & \mathbf{I} & -\mathbf{I}
\end{array}\right] .
$$

Expunging the unit block matrices I in diagonal, we obtain

$$
\mathbf{A} \sim\left[\begin{array}{ccccc}
\mathbf{A}_{0} & & & & \\
\mathbf{A}_{0} & \mathbf{A}_{0} & & & \\
\vdots & & \ddots & & \\
\mathbf{A}_{0} & & & \mathbf{A}_{0} & \\
\mathbf{A}_{0} & & & & \mathbf{A}_{0} \\
0 & -\mathbf{I} & & & \\
0 & 0 & -\mathbf{I} & & \\
\vdots & & \ddots & \ddots & \\
0 & & & 0 & -\mathbf{I}
\end{array}\right] .
$$

Expunging the block matrices $\mathbf{A}_{0}$ in diagonal, we obtain

$$
\mathbf{A} \sim\left[\begin{array}{ccccc}
\mathbf{A}_{0} & & & & \\
\mathbf{A}_{0} & 0 & & & \\
\vdots & & \ddots & & \\
\mathbf{A}_{0} & & & 0 & \\
\mathbf{A}_{0} & & & & 0 \\
0 & -\mathbf{I} & & & \\
0 & 0 & -\mathbf{I} & & \\
\vdots & & \ddots & \ddots & \\
0 & & & 0 & -\mathbf{I}
\end{array}\right] .
$$

Finally, we get

$$
\mathbf{A} \sim\left[\begin{array}{ccccc}
\mathbf{A}_{0} & & & & \\
0 & 0 & & & \\
\vdots & & \ddots & & \\
\dot{0} & & & 0 & \\
0 & & & & 0 \\
0 & -\mathbf{I} & & & \\
0 & 0 & -\mathbf{I} & & \\
\vdots & & \ddots & \ddots & \\
0 & & & 0 & -\mathbf{I}
\end{array}\right] .
$$

As we know, $\operatorname{rank}\left(\mathbf{A}_{0}\right)=t-1$; so

$$
\operatorname{rank}(\mathbf{A})=(t-1) t+t-1=m-1 .
$$

Appendix B: Truncation Errors of Normal Equations at Interior Points

We know that the method of $\mathrm{Su}$ et al. is a discrete approximation to Eq. (4.9), and Eq. (3.7) is a finite 
difference scheme of Poission equation (4.10). For each interior point, according to the Taylor expansion, we have

$$
\begin{aligned}
\frac{w_{i+1}-2 w_{i}+w_{i-1}}{a^{2}}= & \left.\frac{\partial^{2} \mathbf{W}}{\partial y^{2}}\right|_{i}+\left.\frac{a^{2}}{12} \frac{\partial^{4} \mathbf{W}}{\partial y^{4}}\right|_{i}+\left.\frac{a^{4} \partial^{6} \mathbf{W}}{360 \partial y^{6}}\right|_{i} \\
& +\mathbf{O}\left(a^{6}\right), \\
\frac{w_{i-t}-2 w_{i}+w_{i+t}}{a^{2}}= & \left.\frac{\partial^{2} \mathbf{W}}{\partial z^{2}}\right|_{i}+\left.\frac{a^{2}}{12} \frac{\partial^{4} \mathbf{W}}{\partial z^{4}}\right|_{i}+\left.\frac{a^{4} \partial^{6} W}{360 \partial z^{6}}\right|_{i} \\
& +\mathbf{O}\left(a^{6}\right) .
\end{aligned}
$$

Adding the two equations together and omitting the higher terms, we get

$$
\nabla_{a}^{2} \mathbf{W}_{(i)}=\nabla^{2} \mathbf{W}_{(i)}+\frac{a^{2}}{12}\left(\left.\frac{\partial^{4} \mathbf{W}}{\partial y^{4}}\right|_{i}+\left.\frac{\partial^{4} \mathbf{W}}{\partial z^{4}}\right|_{i}\right)+\mathbf{O}\left(a^{4}\right),
$$

where $\nabla^{2}$ is a Laplacian and $\nabla_{a}^{2}$ is the cross difference scheme. From Eqs. (4.2) and (4.9) we have

$$
\nabla^{2} \mathbf{W}=\nabla f_{0}=\nabla\left[\nabla \mathbf{W}_{0}+\mathbf{n}(y, z)\right]=\nabla^{2} \mathbf{W}_{0}+\nabla \mathbf{n}(y, z),
$$

Hence

$$
\nabla^{2}\left(\mathbf{W}-\mathbf{W}_{0}\right)=\nabla \mathbf{n}(y, z),
$$

where $\mathbf{W}_{0}$ is the ideal solution, $\mathbf{W}$ is the actual numerical solution, and $\mathbf{n}(y, z)$ is the gradient error function. For each interior grid point of extended $\boldsymbol{\Omega}_{1}$, let $\mathbf{W}^{\prime}=\mathbf{W}-\mathbf{W}_{0}$. From Eqs. (A10) and (A12), we get

$$
\begin{aligned}
\nabla_{a}{ }^{2} \mathbf{W}_{(i)}^{\prime} & =\nabla^{2} \mathbf{W}_{(i)}^{\prime}+\frac{a^{2}}{12}\left(\left.\frac{\partial^{4} \mathbf{W}^{\prime}}{\partial y^{4}}\right|_{i}+\left.\frac{\partial^{4} \mathbf{W}^{\prime}}{\partial z^{4}}\right|_{i}\right)+\mathbf{O}\left(a^{4}\right) \\
& =\nabla n_{i}+\frac{a^{2}}{12}\left(\left.\frac{\partial^{4} \mathbf{W}^{\prime}}{\partial y^{4}}\right|_{i}+\left.\frac{\partial^{4} \mathbf{W}^{\prime}}{\partial z^{4}}\right|_{i}\right)+\mathbf{O}\left(a^{4}\right) .
\end{aligned}
$$

Hence

$$
\begin{aligned}
& -w_{i+1}^{\prime}-w_{i+t}^{\prime}+4 w_{i}^{\prime}-w_{i-1}^{\prime}-w_{i-t}^{\prime} \\
& =-a^{2} \nabla n_{i}-\frac{a^{4}}{12}\left(\left.\frac{\partial^{4} \mathbf{W}^{\prime}}{\partial y^{4}}\right|_{i}+\left.\frac{\partial^{4} \mathbf{W}^{\prime}}{\partial z^{4}}\right|_{i}\right)+\mathbf{O}\left(a^{6}\right) .
\end{aligned}
$$

\section{Appendix C: Truncation Errors of the Normal Equations at Boundary Points}

1. Derivation of Several Important Formulas

(I) $w_{i}-w_{i+t}=\left.a \frac{\partial \mathbf{W}}{\partial z}\right|_{i+t} ^{i}+\left.\frac{a^{3}}{24} \frac{\partial^{3} \mathbf{W}}{\partial z^{3}}\right|_{i+t} ^{i}+\mathbf{O}\left(a^{5}\right)$.
In the $z$ direction (as shown in Fig. 8), according to Taylor expansion, we have

$$
\begin{aligned}
w_{i+t}= & w_{i}-\left.a \frac{\partial \mathbf{W}}{\partial z}\right|_{i}+\left.\frac{a^{2}}{2 !} \frac{\partial^{2} \mathbf{W}}{\partial z^{2}}\right|_{i}-\left.\frac{a^{3}}{3 !} \frac{\partial^{3} \mathbf{W}}{\partial z^{3}}\right|_{i}+\left.\frac{a^{4}}{4 !} \frac{\partial^{4} \mathbf{W}}{\partial z^{4}}\right|_{i} \\
& +\mathbf{O}\left(a^{5}\right), \\
w_{i-t}= & w_{i}+\left.a \frac{\partial \mathbf{W}}{\partial z}\right|_{i}+\left.\frac{a^{2}}{2 !} \frac{\partial^{2} \mathbf{W}}{\partial z^{2}}\right|_{i}+\left.\frac{a^{3}}{3 !} \frac{\partial^{3} \mathbf{W}}{\partial z^{3}}\right|_{i}+\left.\frac{a^{4}}{4 !} \frac{\partial^{4} \mathbf{W}}{\partial z^{4}}\right|_{i} \\
& +\mathbf{O}\left(a^{5}\right) .
\end{aligned}
$$

Subtract Eq. (A16) from Eq. (A17), divide the result by $2 a$, and we have

$$
\frac{w_{i-t}-w_{i+t}}{2 a}=\left.\frac{\partial \mathbf{W}}{\partial z}\right|_{i}+\left.\frac{a^{2}}{3 !} \frac{\partial^{3} \mathbf{W}}{\partial z^{3}}\right|_{i}+\mathbf{O}\left(a^{4}\right) .
$$

Replace $a$ with $a / 2$, let $\partial \mathbf{W} /\left.\partial z\right|_{i+t} ^{i}$ denote the partial derivative of $\mathbf{W}$ with respect to $z$ at the midpoint between $i$ and $i+t$, and we have

$$
w_{i}-w_{i+t}=\left.a \frac{\partial \mathbf{W}}{\partial z}\right|_{i+t} ^{i}+\left.\frac{a^{3}}{24} \frac{\partial^{3} \mathbf{W}}{\partial z^{3}}\right|_{i+t} ^{i}+\mathbf{O}\left(a^{5}\right) .
$$

Similarly, we have

$$
w_{i+1}-w_{i}=\left.a \frac{\partial \mathbf{W}}{\partial y}\right|_{i} ^{i+1}+\left.\frac{a^{3}}{24} \frac{\partial^{3} \mathbf{W}}{\partial y^{3}}\right|_{i} ^{i+1}+\mathbf{O}\left(a^{5}\right),
$$

(II)

$$
\begin{aligned}
\left.\frac{\partial \mathbf{W}}{\partial z}\right|_{i+t} ^{i}= & \frac{1}{2}\left(\left.\frac{\partial \mathbf{W}}{\partial z}\right|_{i+t}+\left.\frac{\partial \mathbf{W}}{\partial z}\right|_{i}\right)-\left.\frac{a^{2}}{8} \frac{\partial^{3} \mathbf{W}}{\partial z^{3}}\right|_{i+t} ^{i} \\
& -\left.\frac{a^{4}}{384} \frac{\partial^{5} \mathbf{W}}{\partial z^{5}}\right|_{i+t} ^{i}+\mathbf{O}\left(a^{5}\right) .
\end{aligned}
$$

If we set a function $\mathbf{f}(y, z)=\partial \mathbf{W} / \partial z$, then

$$
\left.\frac{\partial \mathbf{W}}{\partial z}\right|_{i+t} ^{i}=\mathbf{f}\left(y, z_{i+t}+\frac{a}{2}\right)=\mathbf{f}\left(y, z_{i}-\frac{a}{2}\right) .
$$

According to the Taylor expansion, omitting the higher terms, we have

$$
\begin{aligned}
\mathbf{f}\left(y, z_{i+t}+\frac{a}{2}\right)= & \mathbf{f}\left(y, z_{i+t}\right)+\left.\frac{a}{2} \frac{\partial \mathbf{f}}{\partial z}\right|_{i+t}+\left.\frac{a^{2}}{8} \frac{\partial^{2} \mathbf{f}}{\partial z^{2}}\right|_{i+t} \\
& +\left.\frac{a^{3}}{8 \times 3 !} \frac{\partial^{3} \mathbf{f}}{\partial z^{3}}\right|_{i+t}+\left.\frac{a^{4}}{16 \times 4 !} \frac{\partial^{4} \mathbf{f}}{\partial z^{4}}\right|_{i+t},
\end{aligned}
$$

$$
\begin{aligned}
\mathbf{f}\left(y, z_{i}-\frac{a}{2}\right)= & \mathbf{f}\left(y, z_{i}\right)-\left.\frac{a}{2} \frac{\partial \mathbf{f}}{\partial z}\right|_{i}+\left.\frac{a^{2}}{8} \frac{\partial^{2} \mathbf{f}}{\partial z^{2}}\right|_{i} \\
& -\left.\frac{a^{3}}{8 \times 3 !} \frac{\partial^{3} \mathbf{f}}{\partial z^{3}}\right|_{i}+\left.\frac{a^{4}}{16 \times 4 !} \frac{\partial^{4} \mathbf{f}}{\partial z^{4}}\right|_{i}
\end{aligned}
$$


Add Eqs. (A23) and (A24), divide the result by 2, and we have

$$
\begin{aligned}
\left.\frac{\partial \mathbf{W}}{\partial z}\right|_{i+t} ^{i}= & \frac{\mathbf{f}\left(y, z_{i+t}\right)+\mathbf{f}\left(y, z_{i}\right)}{2}-\frac{a}{4}\left(\left.\frac{\partial \mathbf{f}}{\partial z}\right|_{i}-\left.\frac{\partial \mathbf{f}}{\partial z}\right|_{i+t}\right) \\
& +\frac{a^{2}}{16}\left(\left.\frac{\partial^{2} \mathbf{f}}{\partial z^{2}}\right|_{i}+\left.\frac{\partial^{2} \mathbf{f}}{\partial z^{2}}\right|_{i+t}\right)-\frac{a^{3}}{96}\left(\left.\frac{\partial^{3} \mathbf{f}}{\partial z^{3}}\right|_{i}-\left.\frac{\partial^{3} \mathbf{f}}{\partial z^{3}}\right|_{i+t}\right) \\
& +\frac{a^{4}}{768}\left(\left.\frac{\partial^{4} \mathbf{f}}{\partial z^{4}}\right|_{i}+\left.\frac{\partial^{4} \mathbf{f}}{\partial z^{4}}\right|_{i+t}\right) .
\end{aligned}
$$

Applying formula (A15) in Eq. (A25), we find

$$
\left.\frac{\partial \mathbf{W}}{\partial z}\right|_{i+t} ^{i}=\frac{1}{2}\left(\left.\frac{\partial \mathbf{W}}{\partial z}\right|_{i}+\left.\frac{\partial \mathbf{W}}{\partial z}\right|_{i+t}\right)-\left.\frac{a^{2}}{8} \frac{\partial^{3} \mathbf{W}}{\partial z^{3}}\right|_{i+t} ^{i}+\mathbf{O}\left(a^{3}\right) .
$$

Apply formulas (A15) and (A26) in Eq. (A25), and we obtain formula (A21). Similarly, we have

$$
\begin{aligned}
\left.\frac{\partial \mathbf{W}}{\partial y}\right|_{i} ^{i+1}= & \frac{1}{2}\left(\left.\frac{\partial \mathbf{W}}{\partial y}\right|_{i+1}+\left.\frac{\partial \mathbf{W}}{\partial y}\right|_{i}\right)-\left.\frac{a^{2}}{8} \frac{\partial^{3} \mathbf{W}}{\partial y^{3}}\right|_{i} ^{i+1} \\
& -\left.\frac{a^{4}}{384} \frac{\partial^{5} \mathbf{W}}{\partial y^{5}}\right|_{i} ^{i+1}+\mathbf{O}\left(a^{5}\right) .
\end{aligned}
$$

2. Truncation Errors of the Normal Equations at Boundary Points of $\boldsymbol{\Omega}_{1}$

The boundary points of $\boldsymbol{\Omega}_{1}$ can be divided into two groups: one group are the corner-boundary points, the others are the line-boundary points.

\section{a. For Corner-Boundary Points}

For example, at point A (as shown in Fig. 4), we have a boundary condition

$$
\left.\frac{\partial \mathbf{W}}{\partial n}\right|_{\partial \Omega_{1}}=\mathbf{g}(y, z)=g_{y} i+g_{z} j .
$$

However,

$$
\begin{aligned}
g_{y}=\left.\frac{\partial \mathbf{W}}{\partial y}\right|_{1} ^{2}= & -\frac{1}{2}\left(\left.\frac{\partial \mathbf{W}}{\partial y}\right|_{1}+\left.\frac{\partial \mathbf{W}}{\partial y}\right|_{2}\right)+\left.\frac{a^{2}}{8} \frac{\partial^{3} \mathbf{W}}{\partial y^{3}}\right|_{1} ^{2} \\
& +\left.\frac{a^{4}}{384} \frac{\partial^{5} \mathbf{W}}{\partial y^{5}}\right|_{1} ^{2}+\mathbf{O}\left(a^{5}\right), \\
g_{z}=\left.\frac{\partial \mathbf{W}}{\partial z}\right|_{1+t} ^{1}= & -\frac{1}{2}\left(\left.\frac{\partial \mathbf{W}}{\partial z}\right|_{1}+\left.\frac{\partial \mathbf{W}}{\partial z}\right|_{1+t}\right)+\left.\frac{a^{2}}{8} \frac{\partial^{3} \mathbf{W}}{\partial z^{3}}\right|_{1+t} ^{1} \\
& +\left.\frac{a^{4}}{384} \frac{\partial^{5} \mathbf{W}}{\partial z^{5}}\right|_{1+t} ^{1}+\mathbf{O}\left(a^{5}\right),
\end{aligned}
$$

and according formulas (A19) and (A20), we get

$$
\begin{gathered}
\left.\frac{\partial \mathbf{W}}{\partial y}\right|_{1} ^{2}=\frac{w_{2}-w_{1}}{a}-\left.\frac{a^{2}}{4 !} \frac{\partial^{3} \mathbf{W}}{\partial y^{3}}\right|_{1} ^{2}+\mathbf{O}\left(a^{4}\right), \\
\left.\frac{\partial \mathbf{W}}{\partial z}\right|_{1+t} ^{1}=\frac{w_{1}-w_{1+t}}{a}-\left.\frac{a^{2}}{4 !} \frac{\partial^{3} \mathbf{W}}{\partial z^{3}}\right|_{1+t} ^{1}+\mathbf{O}\left(a^{4}\right) .
\end{gathered}
$$

Subtract Eq. (A31) from Eq. (A32) and apply formulas (3.3), (3.4), (A29), and (A30) in the subtractive result, omitting the higher terms, and we have

$$
\begin{aligned}
-w_{2}+2 w_{1}-w_{1+t}= & f_{1, t+1}-g_{1,2} \\
& +\frac{a^{3}}{12}\left(\left.\frac{\partial^{3} \mathbf{W}}{\partial y^{3}}\right|_{1} ^{2}-\left.\frac{\partial^{3} \mathbf{W}}{\partial z^{3}}\right|_{t+1} ^{1}\right) \\
& +\frac{a^{5}}{384}\left(\left.\frac{\partial^{5} \mathbf{W}}{\partial y^{5}}\right|_{1} ^{2}-\left.\frac{\partial^{5} \mathbf{W}}{\partial z^{5}}\right|_{t+1} ^{1}\right) .
\end{aligned}
$$

Similarly, at corner points B, C, and D, we have

$$
\begin{gathered}
-w_{t-1}+2 w_{t}-w_{2 t}=f_{t, 2 t}+g_{t-1, t} \\
-\frac{a^{3}}{12}\left(\left.\frac{\partial^{3} \mathbf{W}}{\partial y^{3}}\right|_{t-1} ^{t}+\left.\frac{\partial^{3} \mathbf{W}}{\partial z^{3}}\right|_{2 t} ^{t}\right) \\
-\frac{a^{5}}{384}\left(\left.\frac{\partial^{5} \mathbf{W}}{\partial y^{5}}\right|_{t-1} ^{t}+\left.\frac{\partial^{5} \mathbf{W}}{\partial z^{5}}\right|_{2 t} ^{1 t}\right), \quad \text { (A34) } \\
-w_{m-t+2}+2 w_{m-t+1}-w_{m-2 t+1}=-f_{m-2 t+1, m-t+1} \\
-g_{m-t+1, m-t+2}+\frac{a^{3}}{12}\left(\left.\frac{\partial^{3} \mathbf{W}}{\partial y^{3}}\right|_{m-t+1} ^{m-t+2}+\left.\frac{\partial^{3} \mathbf{W}}{\partial z^{3}}\right|_{m-t+1} ^{m-2 t+1}\right) \\
+\frac{a^{5}}{384}\left(\left.\frac{\partial^{5} \mathbf{W}}{\partial y^{5}}\right|_{m-t+1} ^{m-t+2}+\left.\frac{\partial^{5} \mathbf{W}}{\partial z^{5}}\right|_{m-t+1} ^{1 m-2 t+1}\right), \\
+w_{m-1}-w_{m-t} \quad \text { (A35) } \\
=-f_{m-t, m}+g_{m-1, m}-\frac{a^{3}}{12}\left(\left.\frac{\partial^{3} \mathbf{W}}{\partial y^{3}}\right|_{m-1} ^{m}-\left.\frac{\partial^{3} \mathbf{W}}{\partial z^{3}}\right|_{m} ^{m-t}\right) \\
-\frac{a^{5}}{384}\left(\left.\frac{\partial^{5} \mathbf{W}}{\partial y^{5}}\right|_{m-t} ^{m}-\left.\frac{\partial^{5} \mathbf{W}}{\partial z^{5}}\right|_{m} ^{1 m-t}\right) . \quad \text { (A36) }
\end{gathered}
$$

b. For Points on the $A B$ Boundary Line (Except Points $A$ and $B$ )

From Eq. (A8), we get

$$
w_{i+1}-2 w_{i}+w_{i-1}=\left.a^{2} \frac{\partial^{2} \mathbf{W}}{\partial y^{2}}\right|_{i}+\left.\frac{a^{4}}{12} \frac{\partial^{4} \mathbf{W}}{\partial y^{4}}\right|_{i}+\mathbf{O}\left(a^{6}\right) .
$$

Apply formula (A20) to formula (A37) twice, then replace $\mathbf{W}$ with $\partial \mathbf{W} / \partial y$, and we get

$$
\left.\frac{\partial \mathbf{W}}{\partial y}\right|_{i} ^{i+1}-\left.\frac{\partial \mathbf{W}}{\partial y}\right|_{i-1} ^{i}=\left.a \frac{\partial^{2} \mathbf{W}}{\partial y^{2}}\right|_{i}+\left.\frac{a^{3}}{24} \frac{\partial^{4} \mathbf{W}}{\partial y^{4}}\right|_{i}+\mathbf{O}\left(a^{5}\right) .
$$

Applying formula (A27) to the left-hand side of Eq. (A38), we have

$$
\begin{aligned}
\left.\frac{\partial \mathbf{W}}{\partial y}\right|_{i} ^{i+1}-\left.\frac{\partial \mathbf{W}}{\partial y}\right|_{i-1} ^{i}= & \frac{g_{i, i+1}-g_{i-1, i}}{a}-\left.\frac{a^{3}}{8} \frac{\partial^{4} \mathbf{W}}{\partial y^{4}}\right|_{i} \\
& -\left.\frac{a^{5}}{384} \frac{\partial^{6} \mathbf{W}}{\partial y^{6}}\right|_{i}+\mathbf{O}\left(a^{6}\right) .
\end{aligned}
$$


Therefore from Eqs. (A38) and (A39) we get

$$
\left.a^{2} \frac{\partial^{2} \mathbf{W}}{\partial y^{2}}\right|_{i}=g_{i, i+1}-g_{i-1, i}-\left.\frac{a^{4}}{6} \frac{\partial^{4} \mathbf{W}}{\partial y^{4}}\right|_{i}+\mathbf{O}\left(a^{6}\right) .
$$

Applying formula (A40) in Eq. (A37), we have

$$
w_{i+1}-2 w_{i}+w_{i-1}=g_{i, i+1}-g_{i-1, i}-\left.\frac{a^{4}}{12} \frac{\partial^{4} \mathbf{W}}{\partial y^{4}}\right|_{i}+\mathbf{O}\left(a^{6}\right) .
$$

From the boundary condition given we know that

$$
\begin{aligned}
\left.\frac{\partial \mathbf{W}}{\partial n}\right|_{i}=\left.\frac{\partial \mathbf{W}}{\partial z}\right|_{i+t} ^{i}= & \frac{1}{2}\left(\left.\frac{\partial \mathbf{W}}{\partial z}\right|_{i+t}+\left.\frac{\partial \mathbf{W}}{\partial z}\right|_{i}\right) \\
& -\left.\frac{a^{2}}{8} \frac{\partial^{3} \mathbf{W}}{\partial z^{3}}\right|_{i+t} ^{i}-\left.\frac{a^{4}}{384} \frac{\partial^{5} \mathbf{W}}{\partial z^{5}}\right|_{i+t} ^{i} .
\end{aligned}
$$

From Eqs. (A42) and (A19) we get the following equation:

$$
w_{i}-w_{i+t}=f_{i, i+t}-\left.\frac{a^{3}}{12} \frac{\partial^{3} \mathbf{W}}{\partial z^{3}}\right|_{i+t} ^{i}+\mathbf{O}\left(a^{5}\right) .
$$

Combining Eq. (A43) with Eq. (A41), we get

$$
\begin{array}{r}
-w_{i+1}-w_{i+t}+3 w_{i}-w_{i-1} \\
=f_{i, i+t}-g_{i, i+1}+g_{i-1, i}-\left.\frac{a^{3}}{12} \frac{\partial^{3} \mathbf{W}}{\partial z^{3}}\right|_{i+t} ^{i} \\
+\left.\frac{\mathbf{a}^{4}}{12} \frac{\partial^{4} W}{\partial y^{4}}\right|_{i}+\mathbf{O}\left(a^{5}\right) .
\end{array}
$$

Similarly, for boundary CD, AC, BD (corner points A, $\mathrm{B}, \mathrm{C}$, and $\mathrm{D}$ are not included) we have

$$
\begin{aligned}
-w_{i+1}-w_{i-t} & +3 w_{i}-w_{i-1}=-f_{i, i-t}-g_{i, i+1}+g_{i-1, i} \\
+ & \left.\frac{a^{3}}{12} \frac{\partial^{3} \mathbf{W}}{\partial z^{3}}\right|_{i} ^{i-t}+\left.\frac{a^{4}}{12} \frac{\partial^{4} \mathbf{W}}{\partial y^{4}}\right|_{i}+\mathbf{O}\left(a^{5}\right), \\
-w_{i+1}-w_{i-t} & +3 w_{i}-w_{i+t}=-f_{i-t, i}+f_{i+t, i}-g_{i+1, i} \\
& +\left.\frac{a^{3} \partial^{3} \mathbf{W}}{12 \partial y^{3}}\right|_{i} ^{i+1}+\left.\frac{a^{4}}{12} \frac{\partial^{4} \mathbf{W}}{\partial z^{4}}\right|_{i}+\mathbf{O}\left(a^{5}\right), \quad \\
-w_{i-1}-w_{i-t} & +3 w_{i}-w_{i+t}=-f_{i-t, i}+f_{i+t, i}+g_{i-1, i} \\
& -\left.\frac{a^{3}}{12} \frac{\partial^{3} \mathbf{W}}{\partial y^{3}}\right|_{i-1} ^{i}+\left.\frac{a^{4}}{12} \frac{\partial^{4} \mathbf{W}}{\partial z^{4}}\right|_{i}+\mathbf{O}\left(a^{5}\right) .
\end{aligned}
$$

The above discussion on truncation errors of the normal equations at boundary points are also applicable to the boundary points of $\boldsymbol{\Omega}_{0}$ before extension.

\section{Appendix D: Derivation of Formulation (6.14)}

According to formulation (6.13), we have

$$
\operatorname{lub}_{2}\left(\mathbf{A}^{T} \mathbf{A}\right)=\left[\rho\left(\left[\mathbf{A}^{T} \mathbf{A}\right]^{2}\right)\right]^{1 / 2}=\rho\left(\mathbf{A}^{T} \mathbf{A}\right)=\left[\operatorname{lub}_{2}(\mathbf{A})\right]^{2} .
$$

If $\mathbf{A}^{T} \mathbf{A}$ is invertible, from Eq. (6.11) we obtain

$$
\mathbf{W}^{\prime}=\frac{a^{4}}{24}\left(\mathbf{A}^{T} \mathbf{A}\right)^{-1}\left(\mathbf{M}_{3}-2 \mathbf{M}_{4}\right)+a \cdot\left(\mathbf{A}^{T} \mathbf{A}\right)^{-1} \mathbf{A}^{T} \mathbf{N}^{\prime},
$$

where the first term in the right-hand side of this equation is the wave-front error resulting from the algorithm and the second term is that from the gradient noise. Hence

$$
\begin{aligned}
\left\|\mathbf{W}^{\prime}\right\|_{2} \leq & \frac{a^{4}}{24} \operatorname{lub}_{2}\left[\left(\mathbf{A}^{T} \mathbf{A}\right)^{-1}\right] \mid \mathbf{M}_{3}-2 \mathbf{M}_{4} \|_{2} \\
& +a \operatorname{lub}_{2}\left[\left(\mathbf{A}^{T} \mathbf{A}\right)^{-1} \mathbf{A}^{T}\right] \mid \mathbf{N}^{\prime} \|_{2} \\
= & \frac{a^{4}}{24} \frac{\operatorname{lub}_{2}\left[\left(\mathbf{A}^{T} \mathbf{A}\right)^{-1}\right] \operatorname{lub}_{2}\left(\mathbf{A}^{T} \mathbf{A}\right)}{\operatorname{lub}_{2}\left(\mathbf{A}^{T} \mathbf{A}\right)}\left\|\mathbf{M}_{3}-2 \mathbf{M}_{4}\right\| \\
& +a \frac{\operatorname{lub}_{2}\left[\left(\mathbf{A}^{T} \mathbf{A}\right)^{-1} \mathbf{A}^{T}\right] \operatorname{lub}_{2}(\mathbf{A})}{\operatorname{lub}_{2}(\mathbf{A})}\left\|\mathbf{N}^{\prime}\right\|_{2}, \quad
\end{aligned}
$$

where

$$
\operatorname{cond}\left(\mathbf{A}^{T} \mathbf{A}\right)=\operatorname{lub}_{2}\left[\left(\mathbf{A}^{T} \mathbf{A}\right)^{-1}\right] \operatorname{lub}_{2}\left(\mathbf{A}^{T} \mathbf{A}\right)
$$

is defined as the condition number of normal equations.

If the column rank of matrix $\mathbf{A}$ is full, by employing the Householder transformation, we can find a unitary matrix $\mathbf{P}$ and an invertible upper triangular matrix $\mathbf{R}$ satisfying 17

$$
\mathbf{P A}=\left(\begin{array}{c}
\mathbf{R} \\
0
\end{array}\right), \quad \mathbf{A}=\mathbf{P}^{T}\left(\begin{array}{c}
\mathbf{R} \\
0
\end{array}\right) .
$$

Therefore

$$
\begin{aligned}
\left(\mathbf{A}^{T} \mathbf{A}\right)^{-1} & =\left(\mathbf{R}^{T} \mathbf{R}\right)^{-1}=\mathbf{R}^{-1}\left(\mathbf{R}^{T}\right)^{-1}, \\
\left(\mathbf{A}^{T} \mathbf{A}\right)^{-1} \mathbf{A}^{T} & =\left(\mathbf{R}^{-1} 0\right) \mathbf{P} .
\end{aligned}
$$

According to the definition of matrix norm, i.e., formula (6.13), eliminating the higher terms, we get

$$
\operatorname{lub}_{2}(\mathbf{R})=\operatorname{lub}_{2}(\mathbf{A}), \quad \operatorname{cond}\left(\mathbf{A}^{T} \mathbf{A}\right)=\operatorname{cond}(\mathbf{R})^{2} .
$$

Therefore

$$
\begin{aligned}
\left\|\mathbf{W}^{\prime}\right\|_{2} \leq & \frac{a^{4}}{24} \frac{\operatorname{cond}\left(\mathbf{A}^{T} \mathbf{A}\right)}{\operatorname{lub}_{2}(\mathbf{A})^{2}}\left\|\mathbf{M}_{3}-2 \mathbf{M}_{4}\right\|_{2} \\
& +a \frac{\left[\operatorname{cond}\left(\mathbf{A}^{T} \mathbf{A}\right)\right]^{1 / 2}}{\operatorname{lub}_{2}(\mathbf{A})} \cdot\left\|\mathbf{N}^{\prime}\right\|_{2} .
\end{aligned}
$$

The authors acknowledge stimulating discussions with Dingqiang $\mathrm{Su}$ and Xiangqun Cui of the Nanjing Astronomical Instruments Research Center (NAIRC). This study was supported financially by the Nanjing Branch of the Chinese Academy of Science (NBCAS), and the authors thank Juying Jiang of NBCAS for her enthusiastic help. The authors gratefully acknowledge valuable discussions with Mingchun Zhang and Xi Shen of Southeast University. The authors thank the reviewers of Applied Optics who reviewed this paper. The authors thank James R. Leger at the Electrical and Computer En- 
gineering Department, University of Minnesota, for his useful suggestions and enthusiastic help. The authors also thank Applied Optics copy editor Scott Dineen for his efforts to improve the quality of this paper.

\section{References}

1. W. H. Southwell, "Wave-front estimation from wave-front slope measurements," J. Opt. Soc. Am. 70, 998-1006 (1980).

2. K. R. Freischlad and C. L. Koliopoulos, "Modal estimation of a wave front from difference measurements using the discrete Fourier transform," J. Opt. Soc. Am. 3, 1852-1861 (1986).

3. J. W. Hardy, J. E. Lefebvre, and C. L. Koliopoulos, "Real-time atmospheric compensation," J. Opt. Soc. Am. 67, 360-369 (1977).

4. D. L. Fried, "Least-squares fitting a wave-front distortion estimate to an array of phase-difference measurements," J. Opt. Soc. Am. 67, 370-375 (1977).

5. R. H. Hudgin, "Wave-front reconstruction for compensated imaging," J. Opt. Soc. Am. 67, 375-378 (1977).

6. R. J. Noll, "Phase estimates from slope-type wavefront sensors," J. Opt. Soc. Am. 68, 139-140 (1978).

7. B. R. Hunt, "Matrix formulation of the reconstruction of phase values from phase difference," J. Opt. Soc. Am. 69, 393-399 (1979).

8. J. Hermann, "Least-squares wave front errors of minimum norm,” J. Opt. Soc. Am. 70, 28-35 (1980).

9. F. Roddier and C. Roddier, "Wavefront reconstruction using iterative Fourier transforms," Appl. Opt. 30, 1325-1327 (1991).

10. R. G. Lane and M. Tallon, "Wave-front reconstruction using a Shack-Hartmann sensor," Appl. Opt. 31, 6902-6908 (1992).

11. I. Ghozeil, "Hartmann and other screen tests," in Optical Shop Testing, D. Malacara, ed. (Wiley, New York, 1978).

12. E. T. Pearson, "Hartmann test data reduction," in Advanced
Technology Optical Telescopes IV, L. D. Barr, ed., Proc. SPIE 1236, 628-632 (1990).

13. K. Freischlad, "Wavefront integration from difference data," in Interferometry: Techniques and Analysis, G. M. Brown, O. Y. Kwon, M. Kujawinska, and G. T. Reid, eds., Proc. SPIE 1755, 212-218 (1992).

14. D. Su, S. Jiang, and L. Shao, "A sort of algorithm of wavefront reconstruction for Shack-Hartmann test," in Proceedings of the European Southern Observatory Conference on Progress in Telescope and Instrumentation Technologies, M. H. Ulrich, ed. (European Southern Observatory, Garching, Germany, 1992), pp. 289-292.

15. D. Su, S. Jiang, W. Zou, S. Yang, S. Yang, H. Zhang, and Q. Zhu, "Experiment system of thin-mirror active optics," in Advanced Technology Optical Telescopes V, L. M. Stepp, ed., Proc. SPIE 2199, 609-621 (1994).

16. C. Chen, Equations of Mathematical Physics (China High Educational Press, Beijing, 1992).

17. J. Stoer and R. Bulirsch, Introduction to Numerical Analysis (Springer-Verlag, New York, 1994) (Chinese edition).

18. R. J. Zielinski, B. M. Levine, and B. McNeil, "Hartmann sensors for optical testing," in Optical Manufacturing and Testing II, H. Stahl, ed., Proc. SPIE 3134, 398-406 (1997).

19. W. Zou, "Figure control of large segmented mirror telescope," Master of Science thesis (Nanjing Astronomical Instruments Research Center, Chinese Academy of Sciences, Nanjing, China, 1996), p. 7.

20. B. Jian, "New method for shearing wavefront reconstruction and its applications in wave aberration evaluation," Ph.D. dissertation (Zhejiang University, Hangzhou, China, 1995), p. 6.

21. M. Zhang, Department of Applied Mathematics, Southeast University, Nanjing 210096, China (personal communication, 1999). 